\title{
Upwind Difference Schemes for Hyperbolic Systems of Conservation Laws
}

\author{
By Stanley Osher* and Fred Solomon
}

\begin{abstract}
We derive a new upwind finite difference approximation to systems of nonlinear hyperbolic conservation laws. The scheme has desirable properties for shock calculations. Under fairly general hypotheses we prove that limit solutions satisfy the entropy condition and that discrete steady shocks exist which are unique and sharp. Numerical examples involving the Euler and Lagrange equations of compressible gas dynamics in one and two space dimensions are given.
\end{abstract}

I. Introduction. In this paper we consider numerical solutions of the initial value problem for hyperbolic systems of conservation laws

$$
w_{t}+\sum_{i=1}^{p} f_{i}(w)_{x_{i}}=0, \quad w(x, 0)=\Phi(x),
$$

with $x=\left(x_{1}, \ldots, x_{p}\right)^{T}$, and $-\infty<x_{i}<\infty$ for each $i$.

Here $w(x, t)$ is an $m$-vector of unknowns, and each flux function $f_{i}(w)$ is a vector valued function of $m$ components. The system (1.1) is said to be hyperbolic when all eigenvalues of all nontrivial real linear combinations of the Jacobian matrices $A_{i}(w)=\partial f_{i}(w)$ are real. It is well known that solutions to (1.1) may develop shocks and contact discontinuities, even when the initial data are smooth.

We shall first concentrate on the one-space-dimensional version of (1.1) which we rewrite as

$$
w_{t}+f(w)_{x}=0, \quad w(x, 0)=\Phi(x), \quad-\infty<x<\infty,
$$

and which we assume is strictly hyperbolic-the eigenvalues of $A(w)=\operatorname{grad}_{w} f$ are real and distinct. Multi-dimensional calculations will be done via a dimensional splitting technique discussed below.

Among the numerical methods used to compute discontinuous solutions, those based on shock capturing have been most successful. In this technique the discontinuity is computed as part of the solution. This approach is based on the theorem of Lax and Wendroff [13], which shows that when a finite-difference scheme is in conservation form, then the jump conditions across discontinuities in the limit solution are satisfied automatically. The main advantage of shock capturing lies in its simplicity. The main drawback of most finite-difference schemes is that discontinuities are approximated by continuous transitions that, when narrow, usually

Received November 25, 1980; revised June 4, 1981.

1980 Mathematics Subject Classification. Primary 65M05.

Key words and phrases. Finite difference approximation, upwind schemes, hyperbolic conservation laws.

* Research supported by NSF Research Grant \# MCS 78-0152. 
overshoot or undershoot, or when monotone, usually spread the discontinuity over many grid points.

Another problem, even for certain commonly used schemes, is convergence to nonphysical solutions, e.g., solutions with expansion shocks [1], [8]. The addition of a certain amount of numerical viscosity may remove this difficulty [14] at the cost of spreading also the physical discontinuities.

The numerical method we propose here involves upwind differencing. The idea of upwinding has been around for a long time. Our method will involve only a relatively simple three-point difference approximation to the space derivative in (1.2) and is an extension of an approximation derived by Engquist and Osher [1], [2], [3], for scalar conservation laws. The E-O scheme is in turn related to the Cole-Murman scheme used for the small disturbance equation of transonic flow [17]. Besides its theoretical advantages derived in the references above, recent computations performed at NASA Ames Research Center [7], with an implicit version of E-O, have demonstrated its computational advantages. In particular, very large time steps can be used, significantly reducing the computer time needed to reach steady state, as compared to C-M. Moreover, sharp shock profiles without overshoot were obtained bearing out the theory. Finally, in time-evolving problems, stable, physically correct, results were obtained for $\mathrm{E}-\mathrm{O}$ in many cases where $\mathrm{C}-\mathrm{M}$ went unstable. The time step could be increased by factors of thirty or more.

The success of the E-O scheme for implicit calculations is largely due to the following fact: the numerical flux functions for E-O, viewed as functions of the unknown grid vector, are smoother than those for C-M. The E-O flux functions have Lipschitz continuous partial derivatives. The C-M flux functions are only Lipschitz continuous. Thus, since implicit methods are based on inverting operators using linearization, E-O is naturally much more robust than C-M. We shall expand upon this remark in the next section. However, we note here that the popular upwind scheme of Godunov [6], which like E-O is a monotone scheme, is only as smooth as C-M near the important shock points. Implicit calculations done at U.C.L.A., on simple test problems, indicate much greater robustness for E-O than for the Godunov scheme.

Recently, we learned that Roe, [22], has given a new algorithm for constructing upwind schemes for hyperbolic systems. His algorithm and ours are somewhat related. However, in the scalar case, with $f(u)=u^{2} / 2$, Roe's scheme reduces to $\mathrm{C}-\mathrm{M}$. Thus, in general his scheme possesses stable nonphysical expansion shock solutions. Furthermore it will only be as "smooth" as C-M, making implicit methods with large step sizes problematic.

The difference method we construct here will be given only for explicit one-step time differencing. Given the space differencing, it is fairly routine to extend our scheme to be implicit. We shall discuss this in future work.

Our method is easy to program and we shall show analytically that it yields the following features:

(1) Shocks which satisfy the correct jump conditions (because of conservation form).

(2) Physically correct shocks, i.e., those satisfying an entropy condition.

(3) Exact resolution of steady discontinuities (with at most two grid interior points). 
(4) Uses the smallest number of boundary conditions, i.e., the effect of any nonphysical, (numerical), boundary conditions is minimal.

In addition, the computational evidence indicates that the following property is valid:

(5) (For steady problems) rapid convergence to steady state.

In Section II we review the Engquist-Osher scheme for scalar problems and then construct our new scheme for hyperbolic systems of conservation laws (1.2). Thus the general algorithm is given in that section. We also state the main theorems there.

In Section III we list the algorithms for compressible gas dynamics in both Eulerian and Lagrangian coordinates. It will be seen that our schemes, although simple, are indeed new. They involve various "switches" as described in Sections 2 and 3. A multi-dimensional algorithm based on dimensional splitting is also given there.

In Section IV we will give the results of various computations.

Elsewhere, Engquist and Osher have obtained some global extensions of the main theorems for the potential equation of transonic flow [4]. Together with Mann they are extending this to multi-dimensions [21].

Osher has shown the utility of the scalar E-O scheme in obtaining approximate solutions to a wide class of nonlinear singularly perturbed scalar elliptic boundary value problems [20]. He also has obtained a simple multi-dimensional finite-element version of the scalar E-O scheme, with much theoretical justification, [18]. R. Sanders has proven convergence of the scalar one-dimensional, but variable grid size finite-element generalization of E-O. Reference [19] gives a survey of most of the recent work in this area using the approach of this paper, while [9] gives a rather general survey.

We would like to thank Bram van Leer for his very helpful comments on the first draft of this paper.

II. The General Algorithm and Theoretical Results. We begin by recalling the first order scalar E-O scheme developed in [1], [2]. Consider a nonlinear scalar conservation law in one space variable:

$$
w_{t}+f(w)_{x}=0, \quad w(x, 0)=\Phi(x), \quad-\infty<x<\infty .
$$

The solution $w(x, t)$ is approximated by a mesh function $w_{j}^{n}$ on the mesh $\left\{\left(x_{j}, t^{n}\right)\right\}$ with $x_{j}=j \Delta x, t^{n}=n \Delta t, j=0, \pm 1, \ldots, n=0,1, \ldots$.

The difference approximation in its explicit form, with $w_{j}^{n}$ approximating $w\left(x_{j}, t^{n}\right)$, is

$$
\begin{aligned}
& \text { (a) } w_{j}^{n+1}=w_{j}^{n}-\frac{\Delta t}{\Delta x}\left(\Delta_{+} f_{-}\left(w_{j}^{n}\right)+\Delta_{-} f_{+}\left(w_{j}^{n}\right)\right), \\
& w_{j}^{0}=\Phi\left(x_{j}\right), \quad j=0, \pm 1, \pm 2, \ldots, \\
& \text { (b) } \frac{\Delta t}{\Delta x} \sup \left|f^{\prime}\right|<1 .
\end{aligned}
$$

Here we denote

$$
\Delta_{ \pm} w_{j}= \pm\left(w_{j \pm 1}-w_{j}\right)
$$

and for future use

$$
D_{ \pm} w_{j}=\frac{1}{\Delta x} \Delta_{ \pm} w_{j}
$$


The auxiliary functions $f_{+}$and $f_{-}$are, respectively, the increasing and decreasing parts of $f$.

$$
\begin{aligned}
& \text { (a) } f_{+}(u)=\int_{0}^{u} \chi(s) f^{\prime}(s) d s, \\
& \text { (b) } f_{-}(u)=\int_{0}^{u}(1-\chi(s)) f^{\prime}(s) d s,
\end{aligned}
$$

where $\chi(u)=1 \Leftrightarrow f^{\prime}(u)>0, \chi(u)=0 \Leftrightarrow f^{\prime}(u) \leqslant 0$. Thus, normalizing $f(0)$ to be zero, we have $f=f_{+}+f_{-}$.

When $f$ is convex, the definitions $(2.5)$ reduce to

$$
\begin{aligned}
& \text { (a) } f_{+}(u)=f(\max (u, \bar{u})), \\
& \text { (b) } f_{-}(u)=f(\min (u, \bar{u})),
\end{aligned}
$$

where $\bar{u}$ is the "stagnation" or "sonic" point for which $f^{\prime}(\bar{u})=0$. If $f^{\prime}(u)$ has a fixed sign, e.g., $f^{\prime}>0$ in the region of interest, then the scheme reduces to the classical upwind scheme

$$
w_{j}^{n+1}=w_{j}^{n}-\frac{\Delta t}{\Delta x} \Delta_{-} f\left(w_{j}^{n}\right) \text {. }
$$

Thus the scalar E-O scheme, in regions containing no sonic or shock points, is merely upwind (or downwind) differencing. The flux decomposition appearing in (2.2) gives a recipe for a "switch" in the direction of differencing near sonic or shock points. This "switch" is the basic ingredient of the scheme. See [9], [23] for a survey of other approaches.

The resulting conservation form scheme (2.2)(a) is monotone if the CFL condition (2.2)(b) is valid. This means that viewed as an algorithm

$$
w_{j}^{n+1}=G\left(w_{j+1}^{n}, w_{j}^{n}, w_{j-1}^{n}\right)
$$

the function $G$ is a nondecreasing function of all its arguments.

It has been proven that properties (1)-(4) of the previous section are valid for this scheme. In addition, for initial data in $B V \cap L^{\infty} \cap L^{1}$, the approximate solutions converge with a rate $O\left((\Delta t)^{1 / 2}\right)$ in the $L^{1}$ norm, [11].

We note here that the steady shock property (3) is the one which makes the flux decomposition unique; see [4].

Combining (2.2) with (2.5) enables us to rewrite E-O as

$$
w_{j}^{n+1}=w_{j}^{n}-\frac{\Delta t}{\Delta x}\left[\int_{w_{j-1}^{n}}^{w_{j}^{n}} \chi(w) f^{\prime}(w) d w+\int_{w_{j}^{n}}^{w_{j+1}^{n}}(1-\chi(w)) f^{\prime}(w) d w\right] .
$$

This is the algorithm we shall generalize to systems. Let $\partial f(w)$ be the Jacobian matrix of $f(w)$. We approximate

$$
\partial_{x} f(w) \sim \frac{1}{\Delta x}\left[\int_{w_{j-1}}^{w_{j}} \chi(w) \partial f(w) d w+\int_{w_{j}}^{w_{j+1}}(I-\chi(w)) \partial f(w) d w\right] .
$$

The matrix $\chi(w)$ and the paths of integration remain to be specified.

Let the eigenvalues of $\partial f(w)$ be denoted by $\lambda_{1}(w)<\lambda_{2}(w)<\cdots<\lambda_{m}(w)$ with corresponding right eigenvectors $r_{1}(w), r_{2}(w), \ldots, r_{m}(w)$. Define $T(w)$ to be the 
matrix whose $j$ th column is $r_{j}(w)$. It then follows that

$$
\begin{aligned}
T^{-1}(w) \partial f(w) T(w) & =\Lambda(w)=\operatorname{diag}\left\{\lambda_{k}(w)\right\} \\
& =\left(\begin{array}{cccc}
\lambda_{1}(w) & 0 & \cdots & 0 \\
0 & \lambda_{2}(w) & \cdots & 0 \\
\vdots & & \ddots & \vdots \\
0 & 0 & & \lambda_{m}(w)
\end{array}\right) .
\end{aligned}
$$

We then define

$$
\chi(w)=T(w) \operatorname{diag}\left\{\frac{1}{2}+\frac{1}{2} \operatorname{sign}\left(\lambda_{k}(w)\right)\right\} T^{-1}(w) .
$$

Thus we have

$$
\begin{aligned}
\chi(w) \partial f(w) & =T(w) \operatorname{diag}\left\{\max \left(\lambda_{k}(w), 0\right)\right\} T^{-1}(w)=(\partial f(w))^{+}, \\
(I-\chi(w)) \partial f(w) & =T(w) \operatorname{diag}\left\{\min \left(\lambda_{k}(w), 0\right)\right\} T^{-1}(w)=(\partial f(w))^{-},
\end{aligned}
$$

which defines the "increasing" and "decreasing" fluxes in the vector valued case.

The choice of the paths of integration in (2.9) significantly affects the properties of the scheme. The paths will be chosen in a natural way, so that the preliminary computations needed to construct the scheme are relatively simple. Specifically, construction of the path will be connected to classical techniques for solving the Riemann problem [12], but will be simpler.

We denote the path connecting $w_{j-1}$ to $w_{j}$ by $\Gamma^{j}$ (and of course $\Gamma^{j+1}$ connects $w_{j}$ to $\left.w_{j+1}\right)$. The curve $\Gamma^{j}$ is decomposed into $m$ subcurves

$$
\Gamma^{j}=\bigcup_{k=1}^{m} \Gamma_{k}^{j} \text {. }
$$

These subcurves are related to rarefaction or compression wave solutions of (1.2), and are defined through

$$
\Gamma_{k}^{j}=\left\{\begin{array}{l}
\frac{d w^{(k)}}{d s}=r_{k}\left(w^{(k)}\right) \quad \text { for either } 0 \leqslant s \leqslant s_{k}^{(j)} \text { or } 0 \geqslant s \geqslant s_{k}^{(j)}, \\
w^{(k)}(0)=w^{(k+1)}\left(s_{k+1}^{(j)}\right),
\end{array}\right.
$$

with $w^{(m+1)}\left(s_{m+1}^{(j)}\right)$ defined to be $w_{j-1}$ and $w^{(1)}\left(s_{1}^{(j)}\right)=w_{j}$. In other words, beginning at $w_{j-1}$ we obtain the curve $\Gamma_{m}^{j}$ defined by $w(s)$ such that for $s$ between 0 and $s_{m}$, $d w / d s=r_{m}(w)$, with $w(0)=w_{j-1}$. Then at $s=s_{m}^{(j)}$ we solve $d w / d s=r_{m-1}(w)$ with $w(0)=w^{(m)}\left(s_{m}^{(j)}\right)$. Repeating this procedure, we arrive at an $m$ parameter family of end states $w\left(s_{m}^{(j)}, s_{m-1}^{(j)}, \ldots, s_{1}^{(j)}\right)$. By the implicit function theorem, if $\left|w_{j}-w_{j-1}\right|$ is sufficiently small, there exists exactly one such piecewise smooth curve connecting $w_{j-1}$ to $w_{j}$. This follows since the Jacobian of the mapping $w\left(s_{m}^{(j)}, \ldots, s_{1}^{(j)}\right)$ at the origin is precisely the matrix $\left\{r_{m}\left(w_{j-1}\right), \ldots, r_{1}\left(w_{j-1}\right)\right\}$, which is, of course, nonsingular. (By strict hyperbolicity, it is also clear that the mapping is a smooth function of the $s_{k}$.)

Thus the $\Gamma_{k}^{j}, k=m, \ldots, 1$, locally foliate $R^{m}$, and there is exactly one desired curve $\Gamma^{j}$. 
An important property of this choice of path is that the system "decouples" in the following sense: We have, by (2.14),

$$
\int_{\Gamma_{k}^{j}} \chi(w) \partial f(w) d w=\int_{0}^{s_{k}^{(j)}} \max \left(\lambda_{k}(w(s)), 0\right) r_{k}(w(s)) d s .
$$

Thus the full approximation to the space derivative is

$$
\begin{aligned}
f(w)_{x} \sim \frac{1}{\Delta x}\left[\sum _ { k = 1 } ^ { m } \left(\int_{0}^{s_{k}^{(j)}}\right.\right. & \max \left(\lambda_{k}(w(s)), 0\right) r_{k}(w(s)) d s \\
& \left.\left.+\int_{0}^{s_{k}^{(j+1)}} \min \left(\lambda_{k}(w(s)), 0\right) r_{k}(w(s)) d s\right)\right],
\end{aligned}
$$

and our explicit one-step approximation to (1.2) is

$$
\text { (a) } \begin{aligned}
w_{j}^{n+1}= & w_{j}^{n}-\frac{\Delta t}{\Delta x}\left[\sum _ { k = 1 } ^ { m } \left(\int_{0}^{s_{k}^{(j)}} \max \left(\lambda_{k}(w(s)), 0\right) r_{k}(w(s)) d s\right.\right. \\
& \left.\left.\quad+\int_{0}^{s_{k}^{(j+1)}} \min \left(\lambda_{k}(w(s)), 0\right) r_{k}(w(s)) d s\right)\right] \\
= & w_{j}^{n}-\frac{\Delta t}{\Delta x}\left(\int_{\Gamma^{j}} \chi(w) \partial f(w) d w+\int_{\Gamma^{j+1}}(1-\chi(w)) \partial f(w) d w\right)
\end{aligned}
$$

(b) $\frac{\Delta t}{\Delta x} \sup \left|\lambda_{k}\right|<1$.

It may appear that the ordering of the $\Gamma_{k}^{j}$, for $j$ fixed, is reversed. We begin at $w_{j-1}$ with an $m$ wave and end at $w_{j}$ with a one-wave.

There are natural geometric reasons for this ordering, which we shall discuss in detail in future work. We mention here that this ordering rules out overshoot in the two-point transition region between the constant states of a steady discrete shock, and makes the proof of Theorem 2.2 both possible and simple. This ordering of the paths has the effect of approximating a shock wave by separating the end states a bit in $(x, t)$ space, then inserting a centered compression wave made up of $m, m-1, \ldots, 1$ waves. This full solution is just about to coalesce into a single shock as time evolves in a positive direction.

At first glance the scheme may appear difficult to work with. There are two obvious problems:

First we must find the paths $\Gamma_{k}$, and next we must compute the integrals in closed form. For many physical problems, this procedure is quite simple to implement, as will be seen in Section 3. We proceed by explicitly solving the following equation for each $k$ :

$$
\nabla_{w} \psi \cdot r_{k}(w)=0
$$

and we obtain $(m-1)$ independent solutions, the so-called Riemann invariants. We call them $\psi_{\nu}^{k}, \nu \neq k$. For the equations of gas dynamics to be discussed later, they may be chosen to be simple algebraic functions of the $m$-vector $w$.

We obtain parametric representations for the $\Gamma_{k}$, by requiring that $\psi_{\nu}^{k}(w)$ be constant for each $\nu \neq k$, because

$$
\frac{d}{d s} \psi_{\nu}^{k}(w(s))=\nabla_{w} \psi_{\nu}^{k}(w) \cdot r_{k}(w) \equiv 0
$$


Next we consider the integral (2.15). For many physical equations (again including those discussed in the next section), each $k$ field is either genuinely nonlinear, which means:

$$
\frac{d}{d s} \lambda_{k}(w(s))=\nabla_{w} \lambda_{k}(w(s)) \cdot r_{k}(w(s)) \equiv 1,
$$

or linearly degenerate:

$$
\frac{d}{d s} \lambda_{k}(w(s))=\nabla_{w} \lambda_{k}(w(s)) \cdot r_{k}(w(s)) \equiv 0 .
$$

In the linearly degenerate case, it follows that $\lambda_{k}(w(s))$ is constant on $\Gamma_{k}^{j}$, so

$$
\int_{\Gamma_{k}^{\prime}}(\partial f(w))^{+} d w= \begin{cases}f\left(w^{(k-1)}(0)\right)-f\left(w^{(k)}(0)\right) & \text { if } \lambda_{k}>0, \\ 0 & \text { if } \lambda_{k} \leqslant 0 .\end{cases}
$$

In the genuinely nonlinear case, $\lambda_{k}$ is strictly monotone, decreasing if $s_{k}^{(j)}<0$, increasing if $s_{k}^{(j)}>0$. Thus, there exists at most one point $\bar{s}_{k}^{(j)}$ at which $\lambda_{k}\left(w^{(k)}\left(\bar{s}_{k}^{(j)}\right)\right)$ $=0$. Call this point $\bar{w}_{j}^{(k)}=w^{k}\left(\bar{s}_{k}^{(j)}\right)$, a sonic point. We then have four possibilities:

$$
\int_{\Gamma_{k}^{\prime}}(\partial f(w))^{+} d w=f(w) \mid \begin{array}{ll}
w^{(k-1)}(0) & \text { if } \lambda_{k}\left(w^{(k-1)}(0)\right)>0, \\
\bar{w}_{j}^{k} & \text { if } \lambda_{k}\left(w^{(k+1)}(0)\right) \leqslant 0, \\
w^{(k)}(0) & \text { if } \lambda_{k}\left(w^{(k)}(0)\right)>0, \\
\bar{w}_{j}^{k} & \text { if } \lambda_{k}\left(w^{(k)}(0)\right) \leqslant 0 .
\end{array}
$$

The expressions for $\int_{\Gamma_{k}^{j}}(\partial f(w))^{-} d w$ are analogous and are easily obtained.

Thus the space differencing (2.16), and the resulting one-step approximation (2.17), are easily computed in these very important cases.

Remark 2.1. The difference approximation (2.17) is a first-order accurate conservation form approximation to Eq. (1.2).

Proof. We may rewrite

$$
\begin{aligned}
\int_{\Gamma^{j}} \chi(w) \partial f(w) d w & +\int_{\Gamma^{j+1}}(I-\chi(w)) \partial f(w) d w \\
& =\Delta_{-} f\left(w_{j}\right)+\Delta_{+} \int_{\Gamma^{j}}(I-\chi(w)) \partial f(w) d w \\
& =\Delta_{+} f\left(w_{j}\right)-\Delta_{+} \int_{\Gamma^{j}} \chi(w) \partial f(w) d w \\
& =\Delta_{0} f\left(w_{j}\right)-\frac{1}{2} \Delta_{+} \int_{\Gamma^{j}}|\partial f(w)| d w
\end{aligned}
$$

where $\Delta_{0}=\frac{1}{2}\left(\Delta_{+}+\Delta_{-}\right)$and $|\partial f(w)|=(\partial f(w))^{+}-(\partial f(w))^{-}$.

It is clear that the resulting scheme is in conservation form. To prove first-order accuracy, we must show that

$$
\Delta_{+} \int_{\Gamma^{j}}|\partial f(w)| d w=O\left((\Delta x)^{2}\right)
$$

for smooth functions $w$. 
It is easy to see that

$$
\begin{gathered}
\left.\frac{\partial}{\partial w_{j-1}} \int_{\Gamma^{j}}|\partial f(w)| d w\right|_{w_{j-1}=w_{j}=w}=-|\partial f(w)|, \\
\left.\frac{\partial}{\partial w_{j}} \int_{\Gamma^{j}}|\partial f(w)| d w\right|_{w_{j-1}=w_{j}=w}=|\partial f(w)| .
\end{gathered}
$$

Thus

(2.27) $\Delta_{+} \int_{\Gamma^{j}}|\partial f(w)| d w=-\left|\partial f\left(w_{j}\right)\right| \Delta_{+} \Delta_{-} w_{j}+O\left(\left|\Delta_{+} w_{j}\right|^{2}+\left|\Delta_{-} w_{j}\right|^{2}\right)$.

The result is immediate.

As a consequence of this, and the above-mentioned Lax-Wendroff theorem [13], it follows that, if a sequence of approximate solutions of (2.17) converges boundedly almost everywhere to a limit solution $w(x, t)$, then $w$ is a weak solution of (1.2).

Weak solutions are not in general unique: solutions with expansion shocks are possible. In fact, such solutions do occur in a stable fashion as bounded limits of certain standard difference schemes [1], [7], [8].

In Roe's scheme [22] the integral term in (2.24) is approximated as follows:

$$
\int_{\Gamma^{j}}|\partial f(w)| d w \approx\left|\partial f\left(\tilde{w}_{j}\right)\right| \Delta_{-} w_{j}
$$

where $\tilde{w}_{j}$ is chosen so as to give the equality

$$
\Delta_{-} f\left(w_{j}\right)=\partial f\left(\tilde{w}_{j}\right) \Delta_{-} w_{j} .
$$

In the scalar case with $f(w)=w^{2} / 2$, this is the Cole-Murman switching scheme, which has stable nonphysical shocks. Moreover, the expression on the right of (2.28) is only Lipschitz continuous in $w_{j}, w_{j+1}$, when $w_{j}, w_{j+1}$ are such that $f\left(w_{j}\right)=f\left(w_{j+1}\right)$. The expression on the left has Lipschitz continuous partial derivatives everywhere, including at such points. This explains the superior robustness of E-O for implicit methods, found in [7].

Let us now assume that the strictly hyperbolic system of conservation laws admits an additional convex scalar conservation law. This means there exists a convex scalar function $U(w)$ satisfying an additional conservation law:

$$
\partial_{t} U(w)+\partial x F(w)=0 .
$$

Lax [12] has shown that for such systems the inequality in the sense of distributions,

$$
\partial_{t} U(w)+\partial_{x} F(w) \leqslant 0
$$

is implied for any $w$ which is the limit of solutions of

$$
w_{t}+f(w)_{x}=\varepsilon w_{x x}, \quad \varepsilon>0,
$$

as $\varepsilon \downarrow 0$.

Condition (2.31) is called the entropy condition, or the entropy inequality.

The entropy condition is related to a geometric condition, called the shock condition, which is defined as follows.

Suppose the weak solution $w$ to (1.2) is piecewise continouus at a point of discontinuity. We denote the value of $w$ on the left, respectively right, sides of the 
discontinuity as follows: $w^{L}, w^{R}$. Such a point of discontinuity is a $k$-shock if both

(a) the Rankine-Hugoniot relation

$$
s\left(w^{L}-w^{R}\right)=f\left(w^{L}\right)-f\left(w^{R}\right),
$$

for $s$ the speed of propagation of the shock, holds; and

(b) there are exactly $k-1$ of the characteristic speeds $\lambda_{k}\left(w^{L}\right)<s$ and $m-k$ speeds $\lambda_{k}\left(w^{R}\right)>s$

$$
\lambda_{k-1}\left(w^{L}\right)<s<\lambda_{k}\left(w^{L}\right), \quad \lambda_{k}\left(w^{R}\right)<s<\lambda_{k+1}\left(w^{R}\right) .
$$

This is the shock condition for systems. Lax [12] showed for genuinely nonlinear characteristic fields, that, for weak $k$-shocks, the shock condition is equivalent to the entropy condition

$$
s\left(U\left(w^{L}\right)-U\left(w^{R}\right)\right)-F\left(w^{L}\right)+F\left(w^{R}\right)<0 .
$$

Recently Mock [16] extended this equivalence by removing the hypothesis that the shocks be weak for a class of conservation laws, which includes all scalar convex laws, and a wide class of systems, including all those physical equations of Section III below.

Let $w^{L}$ and $w^{R}$ be the values on the left and right corresponding to a linearly degenerate field. This time $s=\lambda_{k}\left(w^{L}\right)=\lambda_{k}\left(w^{R}\right)$, and the inequalities for such a $k$-contact discontinuity are

$$
\lambda_{k-1}\left(w^{L}\right)<s=\lambda_{k}\left(w^{L}\right)=\lambda_{k}\left(w^{R}\right)<\lambda_{k+1}\left(w^{R}\right) .
$$

It is easy to see that for such fields, for weak $k$-contact discontinuities, the above $k$-contact condition is equivalent to the entropy condition

$$
s\left(U\left(w^{L}\right)-U\left(w^{R}\right)\right)-F\left(w^{L}\right)+F\left(w^{R}\right)=0 .
$$

In order to state our first main theorem, we consider a semidiscrete time continuous approximation to (1.2) of the form

$$
\begin{aligned}
& \frac{\partial}{\partial t} w_{j}=\frac{-1}{\Delta x}\left[\sum _ { k = 1 } ^ { m } \left(\int_{0}^{s_{k}^{(j)}} \max \left(\lambda_{k}(w(s)), 0\right) r_{k}(w(s)) d s\right.\right. \\
&\left.\left.+\int_{0}^{s_{k}^{(j+1)}} \min \left(\lambda_{k}(w(s)), 0\right) r_{k}(w(s)) d s\right)\right] \\
& w_{j}(0)=\Phi\left(x_{j}\right), \quad j=0, \pm 1, \ldots .
\end{aligned}
$$

Our first theorem is

TheOREM 2.1. Suppose $w_{j}(t)$ solves (2.38) and converges boundedly almost everywhere as $\Delta x \rightarrow 0$ to $w(x, t)$. In addition, suppose the quantity $\lim _{\Delta x \rightarrow 0}\left(\sup _{j, t}\left|\Delta_{+} w_{j}(t)\right|\right)$ is sufficiently small and all the fields are genuinely nonlinear. Then the limit solution satisfies the entropy inequality (2.31).

Next we present analytic evidence that our scheme gives excellent shock resolution in the steady case. Let $w^{L}$ and $w^{R}$ be the states on the left and right (of $x=0$, say) for a genuinely nonlinear steady $k$-shock solution of (1.2). Then we seek solutions of (2.17) (or equivalently of (2.38)) which are independent of $n$ (or of $t$ ) and approach $w^{L}$ and $w^{R}$ if $j \rightarrow-\infty$ and $j \rightarrow \infty$, respectively. These are called steady discrete shocks. 
We have

THEOREM 2.2. (i) Existence: Suppose $\left|w^{L}-w^{R}\right|$ is sufficiently small and either (a) $k=1$ or $m$, or more generally (b) there exist $(m-1)$ functions $\psi_{\nu}(w) \in C^{1}(\Omega)$ having linearly independent gradients where the ball $\Omega$ contains $w^{L}, w^{R}$, for $\nu=1, \ldots, m$, $\nu \neq k$, such that $\nabla_{w} \psi_{\nu}(w) \cdot r_{\mu}(w) \equiv 0$ for all $\nu, \mu$ with $1 \leqslant \nu \leqslant k-1, k \leqslant \mu \leqslant m$ and with $k+1 \leqslant \nu \leqslant m, 1 \leqslant \mu \leqslant k$. Then there exists a class of steady discrete $k$-shocks giving a sharp profile in the following sense:

$$
\begin{array}{cc}
w_{j} \equiv w^{L} \quad \text { for } j \leqslant j_{0}-1, \\
w_{j} \equiv w^{R} \quad \text { for } j>j_{0}+1,
\end{array}
$$

with $w_{j_{0}}(\alpha), w_{j_{0}+1}(\alpha)$, a smooth one-parameter family of states separated by a unique sonic point as follows:

(a) $w_{j_{0}}(\alpha)$ is connected to $w^{L}=w_{j_{0}-1}$ via a curve $\Gamma^{j_{0}}$ defined as above, but for which only $\Gamma_{m}^{j_{0}}, \Gamma_{m-1}^{j_{0}}, \ldots, \Gamma_{k}^{j_{0}}$ are used, i.e., $\Gamma_{\nu}^{j_{0}}=\left\{u_{j_{0}}\right\}, \nu=1, \ldots, k-1$, and moreover $\lambda_{\nu} \geqslant 0$ on each $\Gamma_{\nu}^{j_{0}}, \nu=k, k+1, \ldots, m$.

(b) $w_{j_{0}+1}(\alpha)$ is connected to $w^{R}=w_{j_{0}+2}$ via a curve $\Gamma^{j_{0}+2}$ defined as above, but for which only $\Gamma_{k}^{j_{0}+2}, \Gamma_{k-1}^{j_{0}+2}, \ldots, \Gamma_{j}^{j_{0}+2}$ are used, i.e., $\Gamma_{\nu}^{j_{0}+2}=\left\{u_{j_{0}+1}\right\}, \nu=k+1, \ldots, m$, and, moreover, $\lambda_{\nu}(w(s)) \leqslant 0$ on each $\Gamma_{\nu}^{j_{0}+2}, \nu=1,2, \ldots, k$.

(c) The curve $\Gamma^{j_{0}+1}$ has the property that $\lambda_{\nu}(w(s)) \geqslant 0$ on each $\Gamma_{\nu}^{j_{0}+1}, \nu=m, m-$ $1, \ldots, k+1 ; \lambda_{\nu}(w(s)) \leqslant 0$ on each $\Gamma_{\nu}^{j_{0}+1}, \nu=k-1, k-2, \ldots, 1$, and $\lambda_{k}(w(s))$ on $\Gamma_{k}^{j_{0}+1}$ decreases monotonically as $w(s)$ goes from $w_{j_{0}}$ to $w_{j_{0}+1}$. The function $\lambda_{k}(w(s))$ vanishes at $w=\bar{w}$ and $\bar{w}$ depends only on $w^{L}$ and $w^{R}$.

(d) The vector equation

$$
f\left(w_{j_{0}}\right)-f\left(w^{L}\right)+f\left(w_{j_{0}+1}\right)-f(\bar{w})=0
$$

is satisfied by every member of this family.

(ii) Uniqueness. We consider only steady discrete shocks having certain weak monotonicity properties: $\lambda_{\nu}(w)$ on $\Gamma_{\nu}^{j}$ for $\nu \neq k$ does not ever change sign, and if $\lambda_{k}\left(w_{j}\right) \leqslant 0$, then $\lambda_{k}\left(w_{j+1}\right) \leqslant 0$.

Under these circumstances, all steady discrete $k$-shocks are of the form constructed above, if $\sup _{j}\left|w_{j+1}-w_{j}\right|$ is sufficiently small.

We note that, without any of the above restrictions, steady discrete $k$-shocks must be eventually constant, i.e., $w_{j} \equiv w^{L}$ if $j \leqslant a, w_{j} \equiv w^{R}$ if $j \geqslant b$, for some $a, b$. This is the content of Remark 2.3 below.

We also believe that our uniqueness result is valid under weaker hypotheses-see the scalar results in [3], [10].

We remark that the existence of the functions, $\psi_{\nu}$, is assured if there exists a complete set of Riemann invariants. It is also true for the physically interesting examples discussed later.

We also have a result for discrete contacts. In fact, we show that their resolution may be better than for shocks. 
THEOREM 2.3. A class of discrete $k$-contacts exist if $\left|w^{L}-w^{R}\right|$ is sufficiently small. They give an exact profile in the following sense:

Any solution $\left\{w_{j}\right\}_{-\infty}^{\infty}$ with $\lim _{j \rightarrow \infty} w_{j}=w^{R}, \lim _{j \rightarrow-\infty} w_{j}=w^{L}$ which satisfies the equation

$$
w_{j+1}-w_{j}=\int_{w_{j}}^{w_{j+1}} r_{k}(w) d w,
$$

where the kth field is linearly degenerate, and where $\lambda_{k}\left(w^{L}\right)=0$, is an exact solution of (2.17) and (2.38).

It should be noted that the Lagrangian formulation of nonisentropic compressible gas dynamics always has $k=2$ as such a field. The Eulerian formulation has this property if $\pm \infty$ are stagnation points for the flow.

Thus, we have analytic proof of the existence of steady sharp shock profiles. The numerical calculations indicate these shocks are stable (but not the contacts), and this was earlier proven in the scalar case in [3], [10].

We note here that Majda and Ralston [15] have, for a class of first-order conservation form approximations to (1.2), proven the existence of discrete shocks of general speed $s$ such that $(\Delta t / \Delta x) s$ is rational. Modulo some minor technical hypotheses, the principal condition they need, for existence of entropy condition satisfying discrete shock profiles, involves the linearization of the scheme around a constant state $w_{j}=w$ for all $j$. The linearization is obtained by substituting $w_{j}=w+$ $\varepsilon v_{j}$ and collecting terms of order $\varepsilon$.

For our scheme (2.17) this linearization becomes

$$
\begin{aligned}
U_{j}^{n+1}=U_{j}^{n}-\frac{\Delta t}{\Delta x}[(1-\chi(w)) & (\partial f(w))\left(U_{j+1}^{n}-U_{j}^{n}\right) \\
& \left.+\chi(w)(\partial f(w))\left(U_{j}^{n}-U_{j-1}^{n}\right)\right],
\end{aligned}
$$

and the Majda-Ralston condition is automatically valid if the CFL condition (2.17)(b) is true. (However, one of their technical hypotheses is not satisfied for our scheme, but we believe their conclusion to be true, as it was for the E-O scalar case [3]- see also Jennings [10].)

We conclude this section with the following remarks, which are the key to our uniqueness results, and which indicate why discrete steady shock profiles must be sharp.

Remark 2.2. Suppose $w_{j}$ is a solution of (2.17) such that $w_{j_{0}}^{n+1}=w_{j_{0}}^{n}$ and $w_{j_{0}+1}^{n+1}=$ $w_{j_{0}+1}^{n}$ for some $j_{0}$. Moreover, suppose that the eigenvalues $\lambda_{\nu}$ on each $\Gamma_{\nu}^{j}$ for $j=j_{0}$, $j_{0}+1, j_{0}+2$ are nonvanishing and have the same sign for each $j$ (but, in general, different signs for different $\nu$ ). Finally, suppose $\left|w_{j}-w_{j_{0}-1}\right|+\left|w_{j_{0}+1}-w_{j_{0}}\right|+$ $\left|w_{j_{0}+2}-w_{j_{0}+1}\right|$ is sufficiently small. Then $w_{j_{0}}=w_{j_{0}+1}$.

Proof. We have for $j=j_{0}, j_{0}+1$,

$$
\begin{aligned}
0=\sum_{\nu=1}^{m}\left(\int_{0}^{s_{\nu}^{j}} \max \left(\lambda_{\nu}(w(s)), 0\right) r_{\nu}(w(s)) d s\right. \\
\left.+\int_{0}^{s_{\nu}^{j+1}} \min \left(\lambda_{\nu}(w(s)), 0\right) r_{\nu}(w(s)) d s\right) .
\end{aligned}
$$


Let $\nu_{0}$ be such that $\lambda_{\nu_{0}}<0<\lambda_{\nu_{0}+1}$ in all the integrals above. Then, by the mean value theorem, we know that for $j=j_{0}, j_{0}+1$,

$$
\begin{aligned}
0= & \sum_{\nu=\nu_{0}+1}^{m} s_{\nu}^{j} \lambda_{\nu}\left(w_{j}\right)\left(r_{\nu}\left(w_{j}\right)+O\left(\left|s_{\nu}^{j}\right|\right)\right) \\
& +\sum_{\nu=1}^{\nu_{0}} s_{\nu}^{j+1} \lambda_{\nu}\left(w_{j+1}\right)\left(r_{\nu}\left(w_{j+1}\right)+O\left(\left|s_{\nu}^{j+1}\right|\right)\right) .
\end{aligned}
$$

Since the variation in $w_{j}$ is small, the $r_{\nu}+O\left(\left|s_{\nu}\right|\right)$ above are linearly independent. Thus all the factors $s_{\nu}^{j} \lambda_{\nu}\left(w_{j}\right)=0$. Since none of the $\lambda_{\nu}$ vanish, it follows that all the $s_{\nu}^{j}$ vanish. In particular $0=s_{1}^{j_{0}+1}=s_{2}^{j_{0}+1}=\cdots=s_{m}^{j_{0}+1}$, which means $w_{j_{0}}=w_{j_{0}+1}$.

As a simple consequence we have

Remark 2.3. Any discrete $k$-shock must be eventually constant, i.e., there exist integers $a$ and $b$, so that, if $j \leqslant a$, then $w_{j} \equiv w^{L}$, and if $j \geqslant b$, then $w_{j} \equiv w^{R}$.

III. Algorithms for Compressible Gas Dynamics. In this section we apply the general scheme to compressible gas dynamics. As it turns out, the derivations are fairly simple and can be done in closed form. The discussion here will focus on three particular systems although there is ample reason to believe that the scheme can be applied to most of the systems occurring in ideal compressible flow.

We also note that the schemes are constructed below even for large $\left|w_{j}-w_{j-1}\right|$. Our sole restriction is that cavitation does not occur on any of the $\Gamma_{k}^{j}$, i.e., the density always stays positive. This is not unduly restrictive, as our numerical results have shown. However, cavitation does occur when two strong shocks intersect.

A. One-Dimensional Nonisentropic Lagrangian Gas Flow. The equations are

$$
\left[\begin{array}{l}
\tau \\
v \\
E
\end{array}\right]_{t}+\left[\begin{array}{c}
-v \\
p \\
v p
\end{array}\right]_{x}=0
$$

where

$$
p=(\gamma-1) \frac{\left(E-v^{2} / 2\right)}{\tau}
$$

and

$$
\begin{aligned}
& \tau=\text { the specific volume } \\
& v=\text { the velocity } \\
& p=\text { pressure } \\
& E=\text { the energy. }
\end{aligned}
$$

Using the notation of the previous section,

$$
w=\left[\begin{array}{c}
\tau \\
v \\
E
\end{array}\right] \quad \text { and } \quad f(w)=\left[\begin{array}{c}
-v \\
p \\
v p
\end{array}\right]
$$


The Jacobian for this system is

$$
\text { (a) } \partial f(w)=\left[\begin{array}{ccc}
0 & -1 & 0 \\
-\frac{(\gamma-1)}{\tau^{2}}\left(E-\frac{v^{2}}{2}\right) & -(\gamma-1) \frac{v}{\tau} & \frac{\gamma-1}{\tau} \\
-\frac{(\gamma-1)}{\tau^{2}} v\left(E-\frac{v^{2}}{2}\right) & \frac{\gamma-1}{\tau}\left(E-\frac{3 v^{2}}{2}\right) & \frac{(\gamma-1)}{\tau} v
\end{array}\right] \text {, }
$$

and the eigenvalues of $\partial f(w)$ are

$$
\begin{aligned}
\lambda_{2} & =0 \\
\text { (b) } \lambda_{3,1} & = \pm \sqrt{\frac{(\gamma)(\gamma-1)}{\tau^{2}}\left(E-\frac{v^{2}}{2}\right)}
\end{aligned}
$$

The corresponding right eigenvectors are

$$
\begin{gathered}
r_{1}=\left[\begin{array}{c}
1 \\
\sqrt{\frac{(\gamma)(\gamma-1)}{\tau^{2}}\left(E-\frac{v^{2}}{2}\right)} \\
-\frac{(\gamma-1)}{\tau}\left(E-\frac{v^{2}}{2}\right)+v \sqrt{\frac{(\gamma)(\gamma-1)}{\tau^{2}}\left(E-\frac{v^{2}}{2}\right)}
\end{array}\right], \\
r_{2}=\left[\begin{array}{c}
1 \\
0 \\
\left.\frac{\left(E-v^{2} / 2\right)}{\tau}\right]
\end{array}\right] \\
-\sqrt{\frac{(\gamma)(\gamma-1)}{\tau^{2}}\left(E-\frac{v^{2}}{2}\right)} \\
r_{3}=\left[\begin{array}{c}
\gamma-1 \\
-\frac{v^{2}}{\tau}\left(E-\frac{v^{2}}{2}\right)-v \sqrt{\frac{(\gamma)(\gamma-1)}{\tau^{2}}\left(E-\frac{v^{2}}{2}\right)}
\end{array}\right]
\end{gathered}
$$

In view of (2.18) and (2.19), we begin by finding the Riemann invariants for this equation. These are easily obtained:

$$
\begin{array}{ll}
\psi_{2}^{1}=p \tau^{\gamma}, & \psi_{3}^{1}=v+\frac{2}{\gamma-1} \sqrt{\gamma p \tau}=v+\frac{2}{\gamma-1} c, \\
\psi_{1}^{2}=v, & \psi_{3}^{2}=p, \\
\psi_{1}^{3}=p \tau^{\gamma}, \quad \psi_{2}^{3}=v-\frac{2}{\gamma-1} \sqrt{\gamma p \tau}=v-\frac{2}{\gamma-1} c .
\end{array}
$$

We have defined the sound speed: $c=\sqrt{\gamma p \tau}$.

This system of equations has genuinely nonlinear first and third characteristic fields, and linearly degenerate second characteristic field. Thus, from (2.14), (2.16), 
(2.22), and (2.23), we have

$$
\begin{gathered}
\int_{\Gamma^{j}}(\partial f(w))^{+} d w=f\left(w_{j-2 / 3}\right)-f\left(w_{j-1}\right), \\
\int_{\Gamma^{j+1}}(\partial f(w))^{-} d w=f\left(w_{j+1}\right)-f\left(w_{j+2 / 3}\right) .
\end{gathered}
$$

Here we have defined the intersection points

$$
w^{(3)}\left(s_{3}^{(j)}\right)=w_{j-2 / 3}=\Gamma_{3}^{j} \cap \Gamma_{2}^{j} \quad \text { and } \quad w^{(2)}\left(s_{2}^{(j)}\right)=w_{j-1 / 3}=\Gamma_{2}^{j} \cap \Gamma_{1}^{j},
$$

for each $j$.

Moreover,

$$
f\left(w_{j-2 / 3}\right)-f\left(w_{j-1 / 3}\right)=\int_{\Gamma_{2}^{j}} \partial f(w) d w=\int_{\Gamma_{2}^{j}} 0 \cdot r_{2}(w) d w=0 .
$$

Thus, we need only to find either $w_{j-2 / 3}$ or $w_{j-1 / 3}$, and then evaluate $f(w)$ at either point, for each $j$, in order to obtain the difference approximation.

We must find the points of intersection $\Gamma_{3}^{j} \cap \Gamma_{2}^{j}$ defined by (3.8)(a), (b) and $\Gamma_{2}^{j} \cap \Gamma_{1}^{j}$ defined by (3.7), (b), (c):

(a) $\quad p_{j-2 / 3} \tau_{j-2 / 3}^{\gamma}=p_{j-1} \tau_{j-1}^{\gamma}$,

$$
v_{j-2 / 3}-\frac{2}{\gamma-1} \sqrt{\gamma p_{j-2 / 3} \tau_{j-2 / 3}}=v_{j-1}-\frac{2}{\gamma-1} c_{j-1} .
$$

(b) $v_{j-1 / 3}=v_{j-2 / 3}$, $p_{j-1 / 3}=p_{j-2 / 3}$.

(c) $\quad p_{j-1 / 3} \tau_{j-1 / 3}^{\gamma}=p_{j} \tau_{j}^{\gamma}$, $v_{j-1 / 3}+\frac{2}{\gamma-1} \sqrt{\gamma p_{j-1 / 3} \tau_{j-1 / 3}}=v_{j}+\frac{2}{\gamma-1} c_{j}$.

A rather straightforward calculation gives us
(a) $\tau_{j-1 / 3}^{(1-\gamma) / 2}=\left(\frac{(\gamma-1)\left(v_{j}-v_{j-1}\right) / 2+c_{j}+c_{j-1}}{c_{j}\left(1+\left(p_{j-1} / p_{j}\right)^{1 / 2 \gamma}\left(\tau_{j-1} / \tau_{j}\right)^{1 / 2}\right)}\right) \tau_{j}^{(1-\gamma) / 2}$,
(b) $\tau_{j-2 / 3}^{(1-\gamma) / 2}=\left(\frac{(\gamma-1)\left(v_{j}-v_{j-1}\right) / 2+c_{j}+c_{j-1}}{c_{j-1}\left(1+\left(p_{j} / p_{j-1}\right)^{1 / 2 \gamma}\left(\tau_{j} / \tau_{j-1}\right)^{1 / 2}\right)}\right)_{j-1}^{(1-\gamma) / 2}$,
(c) $p_{j-2 / 3}=p_{j-1 / 3}=p_{j-1}\left(\tau_{j-1} / \tau_{j-2 / 3}\right)^{\gamma}$,
(d) $v_{j-2 / 3}=v_{j-1 / 3}=v_{j-1}-\frac{2}{\gamma-1}\left(c_{j-1}-\sqrt{\gamma p_{j-2 / 3} \tau_{j-2 / 3}}\right)$.

Thus we have determined

$$
f\left(w_{j-2 / 3}\right)=\left[\begin{array}{c}
-v_{j-2 / 3} \\
p_{j-2 / 3} \\
(v p)_{j-2 / 3}
\end{array}\right]
$$

in terms of $\tau_{j-2 / 3}$. 
The explicit one-step scheme is

$$
w_{j}^{n+1}=w_{j}^{n}-\frac{\Delta t}{\Delta x}\left(f\left(w_{j-2 / 3}^{n}\right)-f\left(w_{j-1}^{n}\right)+f\left(w_{j+1}^{n}\right)-f\left(w_{j+1 / 3}^{n}\right)\right) .
$$

B. One-Dimensional Nonisentropic Eulerian Gas Flow. The equations are

$$
\begin{aligned}
& \rho_{t}+m_{x}=0 \\
& m_{t}+\left(\frac{m^{2}}{\rho}+(\gamma-1)\left(e-\frac{1}{2} \frac{m^{2}}{\rho}\right)\right)_{x}=0 \\
& e_{t}+\left(\frac{m}{\rho}\left(e+(\gamma-1)\left(e-\frac{1}{2} \cdot \frac{m^{2}}{\rho}\right)\right)\right)_{x}=0
\end{aligned}
$$

where $\rho=$ density, $m=$ momentum, $e=$ specific energy.

Again, using the notation of the previous section,

$$
w=\left[\begin{array}{c}
\rho \\
m \\
e
\end{array}\right] \text { and } f(w)=\left[\begin{array}{c}
\frac{m^{2}}{\rho}+(\gamma-1)\left(e-\frac{1}{2} \frac{m^{2}}{\rho}\right) \\
\frac{m}{\rho}\left(e+(\gamma-1)\left(e-\frac{1}{2} \frac{m^{2}}{\rho}\right)\right)
\end{array}\right]
$$

The Jacobian for this system is

$$
\text { (a) } \partial f(w)=\left[\begin{array}{ccc}
0 & 1 & 0 \\
\frac{\gamma-3}{2} \frac{m^{2}}{\rho^{2}} & (3-\gamma) \frac{m}{\rho} & (\gamma-1) \\
-\frac{\gamma m e}{\rho^{2}}+(\gamma-1) \frac{m^{3}}{\rho^{3}} & \frac{\gamma e}{\rho}-\frac{3(\gamma-1)}{2} \frac{m^{2}}{\rho^{2}} & \frac{\gamma m}{\rho}
\end{array}\right]
$$

and the eigenvalues of $\partial f(w)$ are

(b)

$$
\begin{aligned}
& \lambda_{3,1}=\frac{m}{\rho} \pm \sqrt{(\gamma)(\gamma-1)\left(\frac{e}{\rho}-\frac{m^{2}}{\rho^{2}}\right)}, \\
& \lambda_{2}=\frac{m}{\rho}=u
\end{aligned}
$$

(thus defining the velocity $u$ ).

We also define

$$
\begin{aligned}
& p=(\gamma-1)\left(e-\frac{\rho u^{2}}{2}\right) \\
& c=\sqrt{\frac{\gamma P}{\rho}}
\end{aligned}
$$


The right eigenvectors are

$$
\begin{gathered}
r_{1}=\left[\begin{array}{c}
\frac{m}{\rho}-\sqrt{\frac{(\gamma)(\gamma-1)}{\rho}\left(e-\frac{m^{2}}{2 \rho}\right)} \\
\frac{\gamma e}{\rho}-\frac{(\gamma-1)}{2} \frac{m^{2}}{\rho^{2}}-\frac{m}{\rho} \sqrt{\frac{(\gamma)(\gamma-1)}{\rho}\left(e-\frac{m^{2}}{2 \rho}\right)}
\end{array}\right] \\
r_{2}=\left[\begin{array}{c}
\frac{m}{\rho} \\
\frac{1}{2} \frac{m^{2}}{\rho^{2}}
\end{array}\right], \\
r_{3}=\left[\begin{array}{c}
\frac{m}{\rho}+\sqrt{\frac{(\gamma)(\gamma-1)}{\rho}\left(e-\frac{m^{2}}{2 \rho}\right)} \\
\frac{\gamma e}{\rho}-\frac{(\gamma-1)}{2} \frac{m^{2}}{\rho^{2}}+\frac{m}{\rho} \sqrt{\frac{(\gamma)(\gamma-1)}{\rho}\left(e-\frac{m^{2}}{2 \rho}\right)}
\end{array}\right] .
\end{gathered}
$$

The Riemann invariants for this system are

$$
\begin{array}{ll}
\psi_{2}^{1}=\frac{p}{\rho^{\gamma}}, & \psi_{3}^{1}=u+\frac{2}{\gamma-1} c, \\
\psi_{1}^{2}=u, & \psi_{3}^{2}=p, \\
\psi_{1}^{3}=\frac{p}{\rho^{\gamma}}, & \psi_{2}^{3}=u-\frac{2}{\gamma-1} c .
\end{array}
$$

If we let $u=v, \rho=1 / \tau$, and $e=E / \tau$, we have the same paths of integration as in the preceding Lagrangian formulation. Thus the intersection points are as in (3.9):
(a) $\quad \rho_{j-1 / 3}^{(\gamma-1) / 2}=\left(\frac{(\gamma-1)\left(u_{j}-u_{j-1}\right) / 2+c_{j}+c_{j-1}}{c_{j}\left(1+\left(p_{j-1} / p_{j}\right)^{1 / 2 \gamma}\left(\rho_{j-1} / \rho_{j}\right)^{-1 / 2}\right)}\right) \rho_{j}^{(\gamma-1) / 2}$
(b) $\rho_{j-2}^{(\gamma-1) / 3}=\left(\frac{(\gamma-1)\left(u_{j}-u_{j-1}\right) / 2+c_{j}+c_{j-1}}{c_{j-1}\left(1+\left(p_{j} / p_{j-1}\right)^{1 / 2 \gamma}\left(\rho_{j} / \rho_{j-1}\right)^{-1 / 2}\right)}\right) \rho_{j-1}^{(\gamma-1) / 2}$,
(c) $p_{j-2 / 3}=p_{j-1 / 3}=p_{j-1}\left(\frac{\rho_{j-1}}{\rho_{j-2 / 3}}\right)^{-\gamma}$,
(d) $u_{j-2 / 3}=u_{j-1 / 3}=u_{j-1}-\frac{2}{\gamma-1}\left(c_{j-1}-\sqrt{\frac{\gamma p_{j-2 / 3}}{\rho_{j-2 / 3}}}\right)$.

Again, the first and third fields are genuinely nonlinear, and the second is linearly degenerate. However, all three eigenvectors may have either sign; hence sonic points and switches are defined and used. We shall use the formulation in (2.23) for the second integral, and (2.24) for the first and third. 
First we rewrite $f(w)$, using (3.12)-(3.15),

$$
f(w)=\left[\begin{array}{c}
\rho u \\
\rho u^{2}+p \\
u\left(\frac{\gamma}{\gamma-1} p+\frac{\rho u^{2}}{2}\right)
\end{array}\right] .
$$

Then we have

$$
\int_{\Gamma_{2}^{j}}(\partial f(w))^{+} d w= \begin{cases}f\left(w_{j-1 / 3}\right)-f\left(w_{j-2 / 3}\right) & \text { if } u_{j-2 / 3}>0 \\ 0 & \text { if } u_{j-2 / 3} \leqslant 0\end{cases}
$$

This simplifies to

$$
\int_{\Gamma_{2}^{j}}(\partial f(w))^{+} d w=\left(\rho_{j-1 / 3}-\rho_{j-2 / 3}\right) \max \left(u_{j-2 / 3}, 0\right)\left(\begin{array}{c}
1 \\
u_{j-2 / 3} \\
u_{j-2 / 3}^{2} / 2
\end{array}\right)
$$

and of course

$$
\int_{\Gamma_{2}^{j+1}}(\partial f(w))^{-} d w=\left(\rho_{j+2 / 3}-\rho_{j+1 / 3}\right) \min \left(u_{j+1 / 3}, 0\right)\left(\begin{array}{c}
1 \\
u_{j+1 / 3} \\
u_{j+1 / 3}^{2} / 2
\end{array}\right) .
$$

For the genuinely nonlinear fields, we begin by finding the two "sonic" points, defined as follows.

Let $\bar{w}_{j-1 / 3}$ be that value of $w$ on $\Gamma_{1}^{j}$, or its extension, through $w_{j}$ for which $\lambda_{1}(w)=0$. The second sonic point, $\bar{w}_{j-2 / 3}$, is defined to be that value of $w$ on $\Gamma_{3}^{j}$, or its extension through $w_{j-1}$, for which $\lambda_{3}(w)=0$. Simple calculations give us

(a) $\left(\bar{\rho}_{j-1 / 3}\right)^{(\gamma-1) / 2}=\left(\frac{\gamma-1}{\gamma+1}\right)\left(\frac{u_{j}+2 c_{j} /(\gamma-1)}{c_{j}}\right) \rho_{j}^{(\gamma-1) / 2}$,

(b) $\bar{p}_{j-1 / 3}=p_{j}\left(\bar{\rho}_{j-1 / 3} / \rho_{j}\right)^{\gamma}$,

(c) $\bar{u}_{j-1 / 3}=u_{j}+\frac{2}{\gamma-1}\left(c_{j}-\sqrt{\gamma \bar{p}_{j-1 / 3} / \bar{\rho}_{j-1 / 3}}\right)$;

and
(a) $\left(\bar{\rho}_{j-2 / 3}\right)^{(\gamma-1) / 2}=-\left(\frac{\gamma-1}{\gamma+1}\right)\left(\frac{u_{j-1}-2 c_{j-1} /(\gamma-1)}{c_{j-1}}\right) \rho_{j-1}^{(\gamma-1) / 2}$,
(b) $\bar{p}_{j-2 / 3}=p_{j-1}\left(\bar{\rho}_{j-2 / 3} / \rho_{j-1}\right)^{\gamma}$,
(c) $\bar{u}_{j-2 / 3}=u_{j-1}-\frac{2}{\gamma-1}\left(c_{j-1}-\sqrt{\frac{\gamma \bar{p}_{j-2 / 3}}{\bar{\rho}_{j-2 / 3}}}\right)$.

We now use Eq. (2.24) and the fact that $\lambda_{1}$ is a decreasing function of $\rho$ on $\Gamma_{1}$. This gives us

$$
\int_{\Gamma_{1}^{j}}(\partial f(w))^{+} d w=f(w) \mid \begin{array}{ll}
w_{j} & \text { if } \rho_{j}<\bar{\rho}_{j-1 / 3}, \\
\bar{w}_{j-1 / 3} & \text { if } \rho_{j} \geqslant \bar{\rho}_{j-1 / 3}, \\
w_{j-1 / 3} & \text { if } \rho_{j-1 / 3}<\bar{\rho}_{j-1 / 3}, \\
\bar{w}_{j-1 / 3} & \text { if } \rho_{j-1 / 3} \geqslant \bar{\rho}_{j-1 / 3},
\end{array}
$$


and

$$
\int_{\Gamma_{1}^{j+1}}(\partial f(w))^{-} d w=f(w) \mid \begin{array}{ll}
w_{j+1} & \text { if } \rho_{j+1} \geqslant \bar{\rho}_{j+2 / 3}, \\
\bar{w}_{j+2 / 3} & \text { if } \rho_{j+1}<\bar{\rho}_{j+2 / 3}, \\
w_{j+2 / 3} & \text { if } \rho_{j+2 / 3} \geqslant \bar{\rho}_{j+2 / 3}, \\
\bar{w}_{j+2 / 3} & \text { if } \rho_{j+2 / 3}<\bar{\rho}_{j+2 / 3} .
\end{array}
$$

Since $\lambda_{3}$ is an increasing function of $\rho$ on $\Gamma_{3}^{j},(2.24)$ gives us

$$
\begin{gathered}
\int_{\Gamma_{3}^{j}}(\partial f(w))^{+} d w=f(w) \mid \begin{array}{ll}
w_{j-2 / 3} & \text { if } \rho_{j-2 / 3}>\bar{\rho}_{j-2 / 3}, \\
\bar{w}_{j-2 / 3} & \text { if } \rho_{j-2 / 3} \leqslant \bar{\rho}_{j-2 / 3}, \\
w_{j-1} & \text { if } \rho_{j-1}>\bar{\rho}_{j-2 / 3}, \\
\bar{w}_{j-2 / 3} & \text { if } \rho_{j-1} \leqslant \bar{\rho}_{j-2 / 3} .
\end{array} \\
\int_{\Gamma_{3}^{j+1}}(\partial f(w))^{-} d w=f(w) \mid \begin{array}{ll}
w_{j+1 / 3} & \text { if } \rho_{j+1 / 3} \leqslant \bar{\rho}_{j+1 / 3}, \\
\bar{w}_{j+1 / 3} & \text { if } \rho_{j+1 / 3} \geqslant \bar{\rho}_{j+1 / 3}, \\
w_{j} & \text { if } \rho_{j} \leqslant \bar{\rho}_{j+1 / 3}, \\
\bar{w}_{j+1 / 3} & \text { if } \rho_{j}>\bar{\rho}_{j+1 / 3} .
\end{array}
\end{gathered}
$$

In the last four expressions we use the form (3.19) to define $f(w)$, and (3.18), (3.23), (3.24) to define $w_{j-1 / 3}, \bar{w}_{j-1 / 3}, w_{j-2 / 3}, \bar{w}_{j-2 / 3}$.

We may now use (3.25)-(3.28) to write the explicit version of our scheme:

$$
w_{j}^{n+1}=w_{j}^{n}-\frac{\Delta t}{\Delta x}\left(\sum_{k=1}^{3}\left(\int_{\Gamma_{k}^{j}}(\partial f(w))^{+} d w+\int_{\Gamma_{k}^{j+1}}(\partial f(w))^{-} d w\right)\right) .
$$

C. Two-Dimensional Isentropic Eulerian Gas Flow. The equations are

$$
\begin{aligned}
& \rho_{t}+\sigma_{x}+\tau_{y}=0 \\
& \sigma_{t}+\left(\frac{\sigma^{2}}{\rho}+p\right)_{x}+\left(\frac{\sigma \tau}{\rho}\right)_{y}=0 \\
& \tau_{t}+\left(\frac{\sigma \tau}{\rho}\right)_{x}+\left(\frac{\tau^{2}}{\rho}+p\right)_{y}=0
\end{aligned}
$$

where $\rho=$ density, $\sigma=x$-momentum, $\tau=y$-momentum, $p=k \rho^{\gamma}=$ pressure.

The system is of the form

$$
w_{t}+f(w)_{x}+g(w)_{y}=0 .
$$

We incorporate the method developed in this paper via dimensional splitting and apply successively the following schemes:
(a) $w_{j k}^{n+1 / 2}=w_{j k}^{n}-\frac{\Delta t}{\Delta x}\left(\int_{w_{j k}^{n}}^{w_{j+1, k}^{n}}(\partial f(w))^{-} d w+\int_{w_{j-1, k}^{n}}^{w_{j k}^{n}}(\partial f(w))^{+} d w\right)$,
(b) $w_{j k}^{n+1}=w_{j k}^{n+1 / 2}-\frac{\Delta t}{\Delta y}\left(\int_{w_{j k}^{n+1 / 2}}^{w_{j, k+1}^{n+1}(2}(\partial g(w))^{-} d w+\int_{\left.w_{j, k}^{n+1}\right\}^{2}}^{w_{j k}^{n+1 / 2}}(\partial g(w))^{+} d w\right)$. 
We used dimensional splitting in order to facilitate our computations. In future work concerning implicit time differencing, we shall show its importance. Here we could have as easily done the problem directly.

In order to obtain (3.32)(a), we need the Jacobian

$$
\partial f(w)=\left(\begin{array}{ccc}
0 & 1 & 0 \\
-\frac{\sigma^{2}}{\rho^{2}}+\gamma k \rho^{\gamma-1} & \frac{2 \sigma}{\rho} & 0 \\
-\frac{\sigma \tau}{\rho^{2}} & \frac{\tau}{\rho} & \frac{\sigma}{\rho}
\end{array}\right)
$$

and its eigenvalues and eigenvectors

$$
\begin{aligned}
& \lambda_{1}=\frac{\sigma}{\rho}-\sqrt{\gamma k \rho^{\gamma-1}}=u-c, \\
& \lambda_{2}=\frac{\sigma}{\rho}=u, \\
& \lambda_{3}=\frac{\sigma}{\rho}+\sqrt{\gamma k \rho^{\gamma-1}}=u+c
\end{aligned}
$$

(thus defining $u$ and $c$ ), and

$$
r_{1}=\left(\begin{array}{c}
\frac{1}{\rho}-\sqrt{\gamma k \rho^{\gamma-1}} \\
\frac{\tau}{\rho}
\end{array}\right), \quad r_{2}=\left(\begin{array}{l}
0 \\
0 \\
1
\end{array}\right), \quad r_{3}=\left(\begin{array}{c}
\frac{1}{\rho}+\sqrt{\gamma k \rho^{\gamma-1}} \\
\frac{\tau}{\rho}
\end{array}\right)
$$

The Riemann invariants for this dimensionally split equation are

$$
\begin{array}{ll}
\psi_{2}^{1}=u+\frac{2}{\gamma-1} c, & \psi_{3}^{1}=\frac{\tau}{\rho}=v, \\
\psi_{1}^{2}=c, & \psi_{3}^{2}=u, \\
\psi_{1}^{3}=u-\frac{2}{\gamma-1} c, & \psi_{3}^{2}=v
\end{array}
$$

(thus defining $v$ ).

The first and third fields are genuinely nonlinear, while the second is linearly degenerate. The intersection points are determined by

$$
\begin{gathered}
\sqrt{\kappa \gamma} \rho_{j-1 / 3}^{(\gamma-1) / 2}=c_{j-1 / 3}=c_{j-2 / 3}=\sqrt{\kappa \gamma} \rho_{j-2 / 3}^{(\gamma-1) / 2} \\
=\frac{\gamma-1}{4}\left(u_{j}-u_{j-1}\right)+\frac{1}{2}\left(c_{j}+c_{j-1}\right), \\
u_{j-1 / 3}=u_{j-2 / 3}=\frac{1}{2}\left(u_{j}+u_{j-1}\right)+\frac{1}{\gamma-1}\left(c_{j}-c_{j-1}\right), \\
v_{j-1 / 3}=v_{j}, \quad v_{j-2 / 3}=v_{j-1} .
\end{gathered}
$$


Moreover,

$$
\begin{aligned}
\int_{\Gamma_{2}^{j}}(\partial f(w))^{+} d w & = \begin{cases}f\left(w_{j-1 / 3}\right)-f\left(w_{j-2 / 3}\right) & \text { if } u_{j-2 / 3}>0 \\
0 & \text { if } u_{j-2 / 3} \leqslant 0\end{cases} \\
& =\max \left(u_{j-2 / 3}, 0\right) \rho_{j-2 / 3}\left(\begin{array}{c}
0 \\
0 \\
v_{j}-v_{j-1}
\end{array}\right)
\end{aligned}
$$

and

$$
\int_{\Gamma_{2}^{j+1}}(\partial f(w))^{-} d w=\min \left(u_{j+1 / 3}, 0\right) \rho_{j+1 / 3}\left(\begin{array}{c}
0 \\
0 \\
v_{j+1}-v_{j}
\end{array}\right)
$$

We again define $\bar{w}_{j-2 / 3}, \bar{w}_{j-1 / 3}$ to be the sonic points on $\Gamma_{3}^{j}$ or its extension, and $\Gamma_{1}^{j}$, or its extension, respectively. A simple calculation gives us

$$
\begin{aligned}
\bar{u}_{j-1 / 3} & =\sqrt{\kappa \gamma}\left(\bar{\rho}_{j-1 / 3}\right)^{(\gamma-1) / 2}=\bar{c}_{j-1 / 3}=\frac{\gamma-1}{\gamma+1} u_{j}+\frac{2}{\gamma+1} c_{j}, \\
\bar{v}_{j-1 / 3} & =v_{j}, \\
-u_{j-2 / 3} & =\sqrt{\kappa \gamma}\left(\bar{\rho}_{j-2 / 3}\right)^{(\gamma-1) / 2}=\bar{c}_{j-2 / 3}=-\frac{\gamma-1}{\gamma+1} u_{j-1}+\frac{2}{\gamma+1} c_{j-1}, \\
\bar{v}_{j-2 / 3} & =v_{j-1} .
\end{aligned}
$$

Then, as in (3.25)-(3.28), we have

$$
\int_{\Gamma_{1}^{j}}(\partial f(w))^{+} d w=f(w) \mid \begin{array}{ll}
w_{j} & \text { if } \rho_{j}<\bar{\rho}_{j-1 / 3}, \\
\bar{w}_{j-1 / 3} & \text { if } \rho_{j} \geqslant \bar{\rho}_{j-1 / 3}, \\
w_{j-1 / 3} & \text { if } \rho_{j-1 / 3} \leqslant \bar{\rho}_{j-1 / 3}, \\
\bar{w}_{j-1 / 3} & \text { if } \rho_{j-1 / 3} \geqslant \bar{\rho}_{j-1 / 3},
\end{array}
$$

$$
\int_{\Gamma_{1}^{j+1}}(\partial f(w))^{-} d w=f(w) \mid \begin{array}{ll}
w_{j+1} & \text { if } \rho_{j+1} \geqslant \bar{\rho}_{j+2 / 3}, \\
\bar{w}_{j+2 / 3} & \text { if } \rho_{j+1}<\bar{\rho}_{j+2 / 3}, \\
w_{j+2 / 3} & \text { if } \rho_{j+2 / 3} \geqslant \bar{\rho}_{j+2 / 3}, \\
\bar{w}_{j+2 / 3} & \text { if } \rho_{j+2 / 3}<\bar{\rho}_{j+2 / 3},
\end{array}
$$

$$
\int_{\Gamma_{3}^{j}}(\partial f(w))^{+} d w=f(w) \mid \begin{array}{ll}
w_{j-2 / 3} & \text { if } \rho_{j-2 / 3}>\bar{\rho}_{j-2 / 3}, \\
\bar{w}_{j-2 / 3} & \text { if } \rho_{j-2 / 3} \leqslant \bar{\rho}_{j-2 / 3}, \\
w_{j-1} & \text { if } \rho_{j-1}>\bar{\rho}_{j-2 / 3}, \\
\bar{w}_{j-2 / 3} & \text { if } \rho_{j-1} \leqslant \bar{\rho}_{j-2 / 3},
\end{array}
$$




$$
\int_{\Gamma_{3}}(\partial f(w))^{-} d w=f(w) \mid \begin{array}{ll}
w_{j+1 / 3} & \text { if } \rho_{j+1 / 3} \leqslant \bar{\rho}_{j+1 / 3}, \\
\bar{w}_{j+1 / 3} & \text { if } \rho_{j+1 / 3}>\bar{\rho}_{j+1 / 3}, \\
w_{j} & \text { if } \rho_{j} \leqslant \overline{\boldsymbol{\rho}}_{j+1 / 3}, \\
\bar{w}_{j+1 / 3} & \text { if } \rho_{j}>\overline{\boldsymbol{\rho}}_{j+1 / 3} .
\end{array}
$$

Here we have used (3.36) and (3.39) to define $w_{j-1 / 3}, \bar{w}_{j-1 / 3}, w_{j-2 / 3}$ and $\bar{w}_{j-2 / 3}$.

We insert (3.40)-(3.41) into (3.32)(a) in order to obtain our first splitting step.

The Riemann invariants and eigenvalues for the system approximated in (3.32)(b) are

$$
\begin{array}{lll}
\psi_{2}^{1}=v+\frac{2}{\gamma-1} c, & \psi_{3}^{1}=u, & \lambda_{1}=v-c, \\
\psi_{1}^{2}=c, & \psi_{2}^{3}=v, & \lambda_{2}=v, \\
\psi_{1}^{3}=v-\frac{2}{\gamma-1} c, & \psi_{2}^{3}=u, & \lambda_{3}=v+c .
\end{array}
$$

The intersection points are (using the index $j$ for the discrete $y$ variable):

$$
\begin{array}{r}
\sqrt{\kappa \gamma} \rho_{j-1 / 3}^{(\gamma-1) / 2}=c_{j-1 / 3}=c_{j-2 / 3}=\sqrt{\kappa \gamma} \rho_{j-2 / 3}^{(\gamma-1) / 2} \\
=\frac{\gamma-1}{4}\left(v_{j}-v_{j-1}\right)+\frac{1}{2}\left(c_{j}+c_{j-1}\right), \\
v_{j-1 / 3}=v_{j-2 / 3}=\frac{v_{j}+v_{j-1}}{2}+\frac{1}{\gamma-1}\left(c_{j}-c_{j-1}\right), \\
u_{j-1 / 3}=u_{j}, \quad u_{j-2 / 3}=u_{j-1} .
\end{array}
$$

As usual,

$$
\int_{\Gamma_{2}^{j}}(\partial g(w))^{+} d w= \begin{cases}g\left(w_{j-1 / 3}\right)-g\left(w_{j-2 / 3}\right) & \text { if } v_{j-2 / 3}>0, \\ 0 & \text { if } v_{j-2 / 3} \leqslant 0,\end{cases}
$$

$$
\begin{gathered}
=\max \left(v_{j-2 / 3}, 0\right) \rho_{j-2 / 3}\left(\begin{array}{c}
0 \\
v_{j}-v_{j-1} \\
0
\end{array}\right), \\
\int_{\Gamma_{2}^{j+1}}(\partial g(w))^{-} d w=\min \left(v_{j+1 / 3}, 0\right) \rho_{j+1 / 3}\left(\begin{array}{c}
0 \\
v_{j+1}-v_{j} \\
0
\end{array}\right) .
\end{gathered}
$$

The sonic points are defined by

$$
\begin{aligned}
\bar{v}_{j-1 / 3} & =\sqrt{\kappa \gamma}\left(\bar{\rho}_{j-1 / 3}\right)^{(\gamma-1) / 2}=\bar{c}_{j-1 / 3}=\frac{\gamma-1}{\gamma+1} v_{j}+\frac{2}{\gamma+1} c_{j}, \\
\bar{u}_{j-1 / 3} & =u_{j}, \\
-v_{j-2 / 3} & =\sqrt{\kappa \gamma}\left(\bar{\rho}_{j-2 / 3}\right)^{(\gamma-1) / 2}=\bar{c}_{j-2 / 3}=-\frac{\gamma-1}{\gamma+1} v_{j-1}+\frac{2}{\gamma+1} c_{j-1}, \\
\bar{u}_{j-2 / 3} & =u_{j-1} .
\end{aligned}
$$

Finally, we have the usual expressions for $\int_{\Gamma_{1,3}^{j}}(\partial g(w))^{ \pm} d w$, obtained from (3.45)-(3.48), with $g(w)$ replacing $f(w)$, and the new values of $w_{j-1 / 3}, w_{j-2 / 3}$, $\bar{w}_{j-1 / 3}, \bar{w}_{j-2 / 3}$ replacing those used in the $x$ sweep in (3.40)-(3.43). We substitute into (3.32)(b) and complete the algorithm. 


\section{Proofs of the Main Theorems.}

Proof of Theorem 2.1. We begin by multiplying (2.38) by $\rho\left(x_{j}, t\right) U_{w}\left(w_{j}\right) \Delta x$, with $\rho$ a nonnegative test function having support in $t>0$. We sum over $j$ and add

$$
\rho\left(x_{j}, t\right) \Delta_{+} F\left(w_{j}\right)=\Sigma \rho\left(x_{j}, t\right) \int_{\Gamma_{j+1}} U_{w}(w) \partial f(w) d w
$$

(using the fact that $F_{w}=U_{w} \partial f$ ).

This gives us

$$
\begin{aligned}
\sum \rho\left(x_{j}, t\right) & \Delta x\left(\frac{\partial}{\partial t} U\left(w_{j}\right)+D_{+} F\left(w_{j}\right)\right) \\
= & \sum \rho\left(x_{j}, t\right) \int_{\Gamma_{j+1}}\left(U_{w}(w)-U_{w}\left(w_{j}\right)\right)(\partial f(w))^{-} d w \\
& +\sum \rho\left(x_{j}, t\right) \int_{\Gamma_{j+1}}\left(U_{w}(w)-U_{w}\left(w_{j+1}\right)\right)(\partial f(w))^{+} d w \\
& +\sum\left(\rho\left(x_{j}, t\right)-\rho\left(x_{j+1}, t\right)\right) \int_{\Gamma_{j+1}}\left(U_{w}\left(w_{j+1}\right)\right)(\partial f(w))^{+} d w .
\end{aligned}
$$

Now integrate and sum by parts on the left side of (4.1), and then let $\Delta x \rightarrow 0$. The Lebesgue dominated convergence theorem tells us that the left side of (4.1) approaches $-\iint\left(\rho_{t} U(w)+\rho_{x} F(w)\right) d x d t$. Thus we need only show that the three expressions on the right have a nonpositive sum.

It is easy to see that

$$
\left|\int_{\Gamma_{j+1}} U_{w}\left(w_{j+1}\right)(\partial f(w))^{+} d w\right| \leqslant K\left(u_{j}, u_{j+1}\right)\left|w_{j+1}-w_{j}\right|,
$$

where we shall always denote as $K$ any uniformly bounded nonnegative function on compact sets.

Again, using the Lebesgue dominated convergence theorem, we see that the last term in (4.1) vanishes as $\Delta x \rightarrow 0$.

We now consider the first term above. We may rewrite

$$
U_{w}(w)-U_{w}\left(w_{j}\right)=\int_{\Gamma_{j+1}^{w}} U_{w w}(v) d v
$$

Here the path $\Gamma_{j+1}^{w}$ denotes that part of $\Gamma_{j+1}$ which begins at $w_{j}$ and ends at $w$ (which, we recall, is on $\Gamma_{j+1}$ ).

Thus, as an iterated integral, we may rewrite (dropping obvious superscripts for $\left.s_{\mu}\right)$

$$
\begin{aligned}
\int_{\Gamma_{j+1}}\left(U_{w}(w)-U_{w}\left(w_{j}\right)\right)(\partial f(w))^{-} d w \\
=\sum_{\mu=m}^{1} \sum_{\nu=m}^{\mu+1} \int_{0}^{s_{\mu}}\left(\int_{0}^{s_{\nu}} U_{w w}(w(s)) r_{\nu}(w(s)) d s\right)^{T} \min \left(\lambda_{\mu}(w(t)), 0\right) r_{\mu}(w(t)) d t \\
\quad+\sum_{\mu=m}^{1} \int_{0}^{s_{\mu}}\left(\int_{0}^{t} U_{w w}(w(s)) r_{\mu}(w(s)) d s\right)^{T} \min \left(\lambda_{\mu}(w(t)), 0\right) r_{\mu}(w(t)) d t
\end{aligned}
$$


and similarly

$$
\begin{aligned}
\int_{\Gamma_{j+1}}\left(U_{w}(w)-U_{w}\left(w_{j+1}\right)\right)(\partial f(w))^{+} d w \\
=-\sum_{\mu=m}^{1} \sum_{\nu=1}^{\mu-1} \int_{0}^{s_{\mu}}\left(\int_{0}^{s_{\nu}} U_{w w}(w(s)) r_{\nu}(w(s)) d s\right)^{T} \max \left(\lambda_{\mu}(w(t)), 0\right) r_{\mu}(w(t)) d t \\
\quad-\sum_{\mu=m}^{1} \int_{0}^{s_{\mu}}\left(\int_{t}^{s_{\nu}} U_{w w}(w(s)) r_{\mu}(w(s)) d s\right)^{T} \max \left(\lambda_{\mu}(w(t)), 0\right) r_{\mu}(w(t)) d t
\end{aligned}
$$

Adding the last two equations gives us

$$
\begin{gathered}
\int_{\Gamma_{j+1}}\left(U_{w}(w)-U_{w}\left(w_{j}\right)\right)(\partial f(w))^{-} d w+\int_{\Gamma_{j+1}}\left(U_{w}(w)-U_{w}\left(w_{j+1}\right)\right)(\partial f(w))^{+} d w \\
=\sum_{\mu=m}^{1} \int_{0}^{s_{\mu}} d t\left[\int_{0}^{t} d s\left(U_{w w}(w(t)) r_{\mu}(w(t))\right)^{T} \min \left(\lambda_{\mu}(w(t)), 0\right) r_{\mu}(w(t)) d t\right. \\
\left.\quad+\int_{t}^{s_{\mu}} d s\left(U_{w w}(w(t)) r_{\mu}(w(t))\right)^{T}\left(-\max \left(\lambda_{\mu}(w(t)), 0\right)\right) r_{\mu}(w(t))\right]+R
\end{gathered}
$$

Here the remainder term $R$ simplifies because of the following observation:

$$
\left(r_{l}\right)^{T} U_{w w} r_{k}=0 \text { if } l \neq k .
$$

Proof of 4.7. It was shown in [5] that $U_{w w} \partial f$ is a symmetric matrix. Thus

$$
\lambda_{k} r_{l}^{T} U_{w w} r_{k}=r_{l}^{T} U_{w w}(\partial f) r_{k}=\left((\partial f) r_{l}\right)^{T} U_{w w} r_{k}=\lambda_{l} r_{l}^{T} U_{w w} r_{k}=0
$$

since $\lambda_{k} \neq \lambda_{l}$.

Thus, a typical term in the remainder may be estimated

$$
\begin{aligned}
\mid \int_{0}^{s_{\mu}} d s \int_{0}^{s_{\nu}}\left(U_{w w}(w(s)) r_{\nu}(w(s))-U_{w w}(w(t)) r_{\nu}(w(t))\right)^{T} \\
\quad \times \min \left(\lambda_{\mu}(w(t)), 0\right) r_{\mu}(w(t)) d t \mid \\
\leqslant K\left(\left|s_{\mu}\right|+\left|s_{\nu}\right|\right)\left|s_{\mu}\right|\left|s_{\nu}\right| \sup \left|\lambda_{\mu}\right|
\end{aligned}
$$

while the other terms in (4.6) can easily be shown to be estimable by

$$
-K \sum_{\mu} \inf \left|\lambda_{\mu}\right| s_{\mu}^{2}
$$

Thus, if none of the eigenvalues $\left|\lambda_{\mu}(w(t))\right|$ vanish in a neighborhood of length $O\left(s_{\mu}\right)$ of the appropriate integrand, the expression on the right of (4.6) is negative, given sufficiently small $\left|\Delta_{+} w_{j}\right|$.

Suppose, on the other hand, $\mu_{0}$ is a genuinely nonlinear field and $\lambda_{\mu_{0}}$ vanishes within length $O\left(\left|s_{\mu_{0}}\right|\right)$ of the appropriate integrand. Then the first terms in (4.6) are estimable by

$$
-K \sum_{\mu \neq \mu_{0}} \inf \left|\lambda_{\mu}\right| s_{\mu}^{2}-K_{\mu_{0}}\left|s_{\mu_{0}}^{3}\right|
$$

Thus, the desired inequality is of the form

$$
\left|s_{\mu_{0}}^{3}\right|+c_{1} s_{\mu}^{2} \geqslant c_{2}\left(s_{\mu}^{2}\left|s_{\mu_{0}}\right|+\left|s_{\mu}\right| s_{\mu_{0}}^{2}\right)
$$


for $c_{1}, c_{2}$ positive and fixed, and $0 \leqslant\left|s_{\mu}\right|,\left|s_{\mu_{0}}\right|<\varepsilon$ for some $\varepsilon$ sufficiently small. But the inequality (4.11) is valid unless both

$$
\begin{aligned}
& \text { (a) } s_{\mu_{0}}^{2} \leqslant c_{2}\left(s_{\mu}^{2}+\left|s_{\mu}\right|\left|s_{\mu_{0}}\right|\right), \\
& \text { (b) }\left|s_{\mu}\right| \leqslant \frac{c_{2}}{c_{1}} s_{\mu_{0}}^{2} /\left(1-\frac{c_{2}}{c_{1}}\left|s_{\mu_{0}}\right|\right) .
\end{aligned}
$$

It is easy to see that for sufficiently small $\varepsilon$ these inequalities are incompatible.

Proof of Theorem 2.1. The following equations are easily seen to be equivalent to the existence of a steady discrete $k$-shock solution to (2.38) of the type defined in (2.39)

$$
\begin{aligned}
& \text { (a) } 0=\int_{\Gamma_{j_{0}}}(\partial f(w))^{-} d w, \\
& \text { (b) } 0=\int_{\Gamma_{j_{0}}}(\partial f(w))^{+} d w+\int_{\Gamma_{j_{0}+1}}(\partial f(w))^{-} d w, \\
& \text { (c) } 0=\int_{\Gamma_{j_{0}+1}}(\partial f(w))^{+} d w+\int_{\Gamma_{j_{0}+2}}(\partial f(w))^{-} d w, \\
& \text { (d) } 0=\int_{\Gamma_{j_{0}+2}}(\partial f(w))^{+} d w .
\end{aligned}
$$

These four equations are not independent. If any three are valid, then the fourth follows automatically. In general, if we have a steady solution, except perhaps at one point $j=j_{1}$, to any consistent approximation in conservation form to (1.2) satisfying $w_{j} \rightarrow w^{L}, w^{R}$ as $j \rightarrow-\infty, \infty$ with $f\left(w^{L}\right)=f\left(w^{R}\right)$, then the steady equation is also valid at that point $j=j_{1}$; see [10].

We must merely verify any three of (4.13)(a)-(d).

Now we require $w_{j_{0}}$ to be any state which can be reached from $w^{L}$ via an $m$ to $k$ wave along a path of the type $\Gamma_{j_{0}}=\cup_{\nu=1}^{m} \Gamma_{\nu}^{j_{0}}$ mentioned in (2.13), (2.14), with the restriction that $\Gamma_{1}^{j_{0}}=\Gamma_{2}^{j_{0}}=\Gamma_{k-1}^{j_{0}}=\left\{w_{j_{0}}\right\}$. This gives us an $(m-k+1)$-parameter family of possible end states $w_{j_{0}}\left(s_{m}^{j_{0}}, s_{m-1}^{j_{0}}, \ldots, s_{k}^{j_{0}}\right)$. If the $\left|s_{\nu}^{j_{0}}\right|$ are sufficiently small, then (4.13)(a) is automatically valid, since each $\lambda_{\nu}$, for $\nu=k, \ldots, m$, is nonnegative on $\Gamma_{v}^{j_{0}}$.

We next require $w_{j_{0}+1}$ to be any state which can be reached from $w^{R}$ via a 1 to $k$ wave along a path of the type $\Gamma_{j_{0}+2}=\bigcup_{\nu=1}^{m} \Gamma_{\nu}^{j_{0}+2}$ with the restriction that $\Gamma_{m}^{j_{j}+2}=$ $\Gamma_{m-1}^{j_{0}+2}=\cdots=\Gamma_{k+1}^{j_{0}+2}=w_{j_{0}+1}$. This gives us a $k$-parameter family of possible end states $w_{j_{0}+1}\left(s_{k}^{j_{0}+2}, \ldots, s_{1}^{j_{j}+2}\right)$. Again, if the $\left|s_{\nu}^{j_{0}+2}\right|$ are sufficiently small, then (4.13)(d) is automatically valid since each $\lambda_{\nu} \leqslant 0$ on $\Gamma_{\nu}^{j_{0}+2}$ for $\nu=1, \ldots, k$.

Finally we connect $w_{j_{0}}$ to $w_{j_{0}+1}$ along $\Gamma_{j_{0}+1}$. We claim to be able to do this in such a way that each $\lambda_{\nu}$ is of one sign on $\Gamma_{\nu}^{j_{0}+1}$ except $\lambda_{k}$ which changes sign on $\Gamma_{k}^{j^{+1}}$. This sign change occurs only once, by genuine nonlinearity. To prove this, for small $\left|w^{L}-w^{R}\right|$, we note that $w^{L}$ is connected to $w^{R}$ along a $k$ shock, and, by Lax [12], the curve of $k$ rarefactions through $u^{L}$ has second-order contact with the curve of $k$-shocks. Thus equation (4.13)(b) becomes

$$
0=f\left(w_{j_{0}}\right)-f\left(w^{L}\right)+f\left(w_{j_{0}+1}\right)-f(\bar{w}),
$$

where $\bar{w}$ is the unique (sonic) point at which $\lambda_{k}(u)$ vanishes on $\Gamma_{k}^{j_{0}+1}$. 
There are two obvious solutions to (4.14) subject to the above conditions. One is $w_{j_{0}}=w^{L}, w_{j_{0}+1}=\bar{w}$, and the second is $w_{j_{0}}=\bar{w}, w_{j_{0}+1}=w^{R}$. Either solution corresponds to the situation where $w^{L}$ is connected to $w^{R}$ by a single curve of the usual type. Moreover the eigenvalues $\lambda_{\nu}$ are all of one sign on the curves $\Gamma_{\nu}$, except $\lambda_{k}$ which vanishes at the unique point $\bar{w}$ on $\Gamma_{k}$. Then the single point of transition is either $w_{j_{0}+1}$ or $w_{j_{0}}$.

Now we claim that the sonic point $\bar{w}$ depends only on $w^{L}$ and $w^{R}$, not on the choice of $w_{j_{0}}$ and $w_{j_{0}+1}$.

To prove this, we use our hypothesis and define $m-1$ functions with linearly independent gradients in a neighborhood containing $w^{L}$ and $w^{R}$, as follows. Let $\varphi_{k+1}(w), \ldots, \varphi_{m}(w)$ be such that $\nabla_{w} \varphi_{\nu} \cdot r_{\mu} \equiv 0$ if $1 \leqslant \mu \leqslant k$ and $\varphi_{1}(w), \ldots, \varphi_{k-1}(w)$ satisfy $\nabla_{w} \varphi_{\nu} \cdot r_{\mu}(w) \equiv 0$ if $k \leqslant \mu \leqslant m$. Let $w$ be any point on $\Gamma_{k}^{j_{0}+1}$.

Then $\varphi_{\nu}\left(w^{L}\right) \equiv \varphi_{\nu}(w)$ for $\nu=1, \ldots, k-1$, and $\varphi_{\nu}\left(w^{R}\right) \equiv \varphi_{\nu}(w)$ for $\nu=k+$ $1, \ldots, m$. These two manifolds intersect along a curve containing $\Gamma_{k}^{j^{+}+1}$. This curve thus depends only on $w^{L}$ and $w^{R}$.

Along $\Gamma_{k}^{j_{0}+1}$, we have $d \lambda_{k}(w(s)) / d s=\nabla_{w} \lambda_{k} \cdot r_{k} \neq 0$, so $\bar{w}$, the sonic point, is uniquely determined.

To connect $w_{j_{0}}$ to $w_{j_{0}+1}$ along $\Gamma^{j_{0}+1}$ we obtain an equation

$$
\begin{aligned}
0= & w_{j_{0}+1}\left(s_{m}^{j_{0}}, s_{m-1}^{j_{0}}, \ldots, s_{k}^{j_{0}} ; s_{m}^{j_{0}+1}, s_{m-1}^{j_{0}+1}, \ldots, s_{1}^{j_{0}+1}\right) \\
& -w_{j_{0}+1}\left(s_{k}^{j_{0}+2}, \ldots, s_{1}^{j_{0}+2}\right) .
\end{aligned}
$$

Consider (4.14) and (4.15) together. This gives us a system of $2 m$ equations in $2 m+1$ unknowns. We view $s_{k}^{j_{0}+2}$ as the independent variable.

We can write the system of equations as

$$
H\left(s_{m}^{j_{0}}, s_{m-1}^{j_{0}}, \ldots, s_{k}^{j_{0}} ; s_{m}^{j_{0}+1}, s_{m-1}^{j_{0}+1}, \ldots, s_{1}^{j_{0}+1} ; s_{k-1}^{j_{0}+2}, \ldots, s_{1}^{j_{0}+2} ; s_{k}^{j_{0}+2}\right)=0
$$

One solution is obtained by taking all the above $s_{\nu}^{j_{0}}$ to be zero which corresponds to letting $w_{j_{0}}=w^{L}$, and fixing $w_{j_{0}+1}=\bar{w}$, which corresponds to letting $s_{1}^{j_{0}+1}=$ $s_{2}^{j_{0}+1}=\cdots=s_{k-1}^{j_{0}+1}=0$. Also $\bar{s}_{m}^{j_{0}+1}, \ldots, \bar{s}_{k}^{j_{0}+1}$, and $s_{k}^{j_{0}+2}, \ldots, s_{1}^{j_{0}+2}$ are fixed so that $\bar{w}$ is connected to $w^{R}$ at these values $\bar{s}_{k}^{j_{0}+2}, \ldots, \bar{s}_{1}^{j_{0}+2}$.

By the implicit function theorem, we need only show that the differential of $H$ with respect to the $2 m$ dependent variables is invertible at this fixed point. It is easy to see that the $2 m \times 2 m$ matrix of the differential is

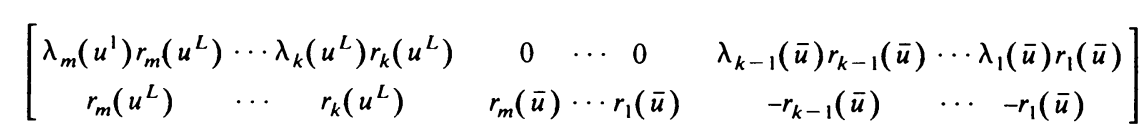

and hence is invertible.

Thus we have proven existence of the one-parameter family of steady discrete shocks.

The proof of the part of this theorem just completed, depends heavily on the ordering in $k$ of the subcurves, $\Gamma_{k}^{j}$. We shall show in the future that a reordering must lead to overshoot. Here we merely show the difficulties which would occur in the preceding proof if we do reorder the curves. We take the case $m=2$, as an example. The only possible reordering is a reversal: $\Gamma_{j}^{j}$ goes through $w_{j-1}, \Gamma_{2}^{j}$ through $w_{j}$. 
Let $w^{L}, w^{R}$, be the left and right states for a weak one-shock. In the proof just completed, we connected $w^{L}$ to $w^{R}$ via a (small) two-wave and then a (relatively large) one-wave, so that the sonic point $\bar{w}$ lies on the one-wave. A steady discrete shock, with one point of transition $\bar{w}=w_{j_{0}}$, was then shown to exist. Finally, a perturbation argument gave us our desired result. Suppose we proceed analogously with our new path, connecting $w^{L}$ to $w^{R}$ along a one- and a two-wave, with $\bar{w}$, the new sonic point, lying on the one-wave. We cannot, in general, let any point on this composite wave act as $w_{j_{0}}$. For if $w_{j_{0}}$ lies on the two-wave, then (4.13)(c) implies that $f\left(w^{R}\right)=f\left(w_{j_{0}}\right)$, which is false. If it lies anywhere else on the wave, then (4.13)(c), the mean value theorem for integrals, and the linear independence of the eigenvectors $r_{1}$ and $r_{2}$, imply that $w^{L}$ is connected to $w^{R}$ by a single one-compression wave. This is false in general, and false for the equations of compressible gas flow.

This argument shows that the proof just completed would become more difficult with any reordering.

Next we discuss the uniqueness of these steady, discrete, shocks. Remark 2.3 tells us that there exist integers $a$ and $b$ such that, if $j \leqslant a$, then $w_{j} \equiv w^{L}$, and if $j \geqslant b$, then $w_{j} \equiv w^{R}$.

Let $j_{0}$ be such that $\lambda_{k}\left(w_{j}\right)>0$ for $j \leqslant j_{0}$, and $\lambda_{k}\left(w_{j_{0}+1}\right) \leqslant 0$. It follows that $w_{j} \equiv w^{L}$ for $j \leqslant j_{0}-1$. Then the first nontrivial equation to be verified is

$$
0=\int_{\Gamma^{j} j_{0}}(\partial f(w))^{-} d w=\sum_{\nu=1}^{k-1} \int_{0}^{s_{\nu}^{j_{0}}} \min \left(\lambda_{\nu}(w(s)), 0\right) r_{\nu}(w(s)) d s .
$$

By the mean value theorem for integrals, it follows that $s^{j_{0}}=s_{2}^{j_{0}}=\cdots=s_{k-1}^{j_{0}}$, and hence $w_{j_{0}}$ is connected to $w^{L}$ along an $m$ to $k$ wave as described in the construction of discrete shocks. The next equation to be verified then becomes

$$
\begin{aligned}
0 & =f\left(w_{j_{0}}\right)-f\left(w^{L}\right)+\sum_{\nu=1}^{k} \int_{0}^{s_{\nu}^{j_{\nu}+1}} \min \left(\lambda_{\nu}(w(s)), 0\right) r_{\nu}(w(s)) d s \\
& =f\left(w_{j_{0}}\right)-f\left(w^{L}\right)+f\left(w_{j_{0}+1}\right)-f(\bar{w}),
\end{aligned}
$$

with $\bar{w}$ the unique point on the $k$ wave at which $\lambda_{k}$ vanishes.

By our hypothesis and the remark above, it follows that $w_{j} \equiv w^{R}$ for $j \geqslant j_{0}+2$. Thus the last nontrivial equation to be verified is

$$
0=\sum_{\nu=k+1}^{m} \int_{0}^{s_{\nu}^{j_{0}+2}} \max \left(\lambda_{\nu}(w(s)), 0\right) r_{\nu}(w(s)) d s,
$$

which implies in the usual fashion that $w_{j_{0}+1}$ is connected to $w^{R}$ via a $k$ to 1 wave, as in the proof of existence.

Thus all solutions of this type solve (4.16).

Proof of Theorem 2.3. The following equations are easily seen to be equivalent to the existence of steady discrete contact discontinuity solutions of the desired type. For each $j$,

$$
\begin{aligned}
0 & =\int_{\Gamma^{j}}(\partial f(w))^{+} d w+\int_{\Gamma^{j+1}}(\partial f(w))^{-} d w \\
& =\int_{\Gamma_{k}^{j}} \max \left(\lambda_{k}(w), 0\right) r_{k}(w) d w+\int_{\Gamma_{k}^{j+1}} \min \left(\lambda_{k}(w), 0\right) r_{k}(w) d w .
\end{aligned}
$$

This is valid, since $\lambda_{k} \equiv 0$, as a consequence of our hypothesis. 
V. Numerical Examples. Numerical results were obtained through computer implementation on the UCLA IBM 3033. We first discuss results for 0-speed discontinuities. We have three examples:

1. (Figure 1) 0-speed shock-Euler equations 1-D nonisentropic ( $\rho$ component).

2. (Figure 2) 0-speed contact-Euler equations 1-D nonisentropic ( $\rho$ component).

3. (Figure 3 ) 0 -speed contact-Lagrange equations 1-D nonisentropic ( $\tau$ component).

Although the theory of steady discontinuities given above was only local, our scheme was successful for large discontinuities, as seen below.

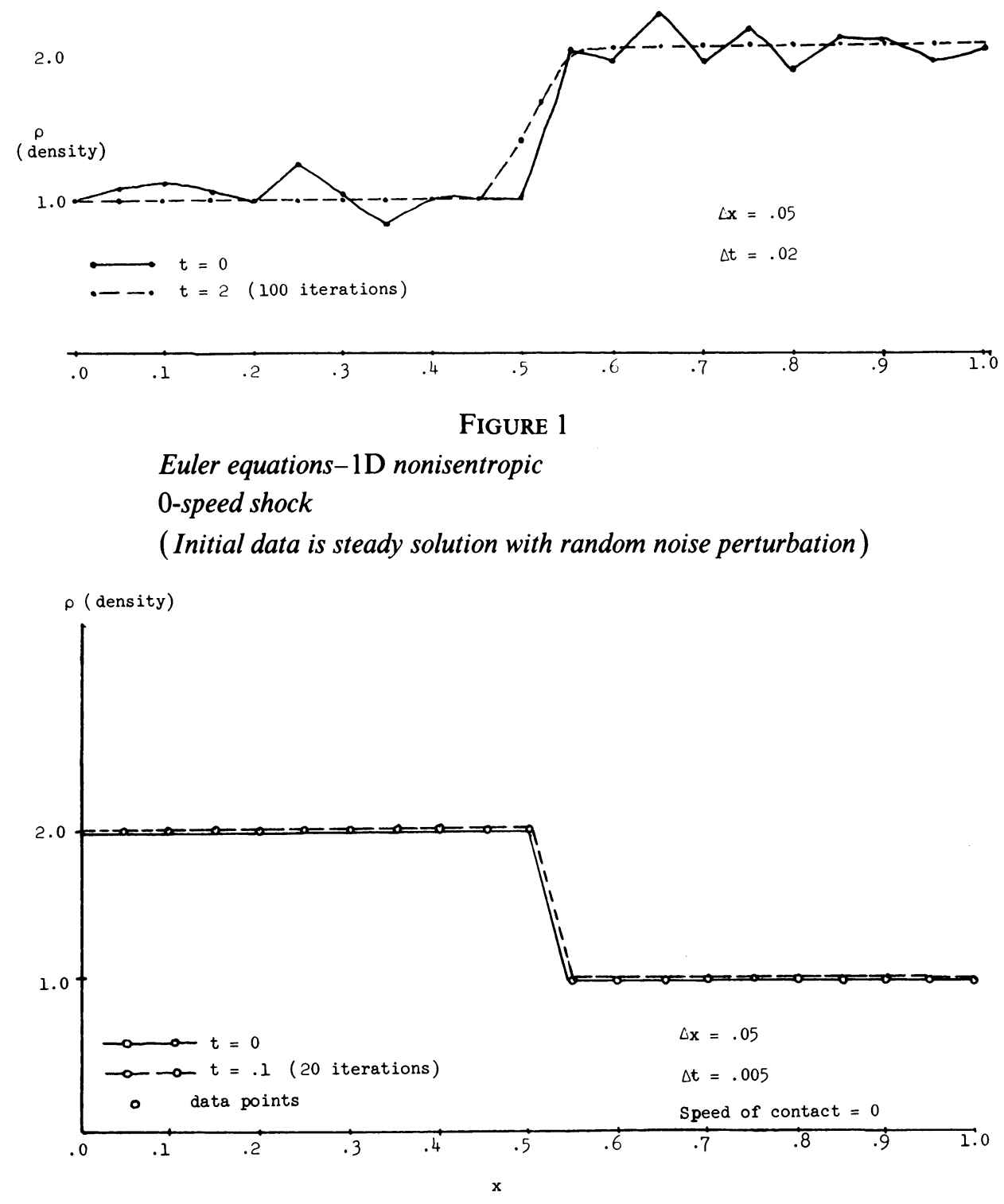

FIGURE 2

Euler equations-1D nonistentropic

0 -speed contact discontinuity

(Initial data is steady solution) 


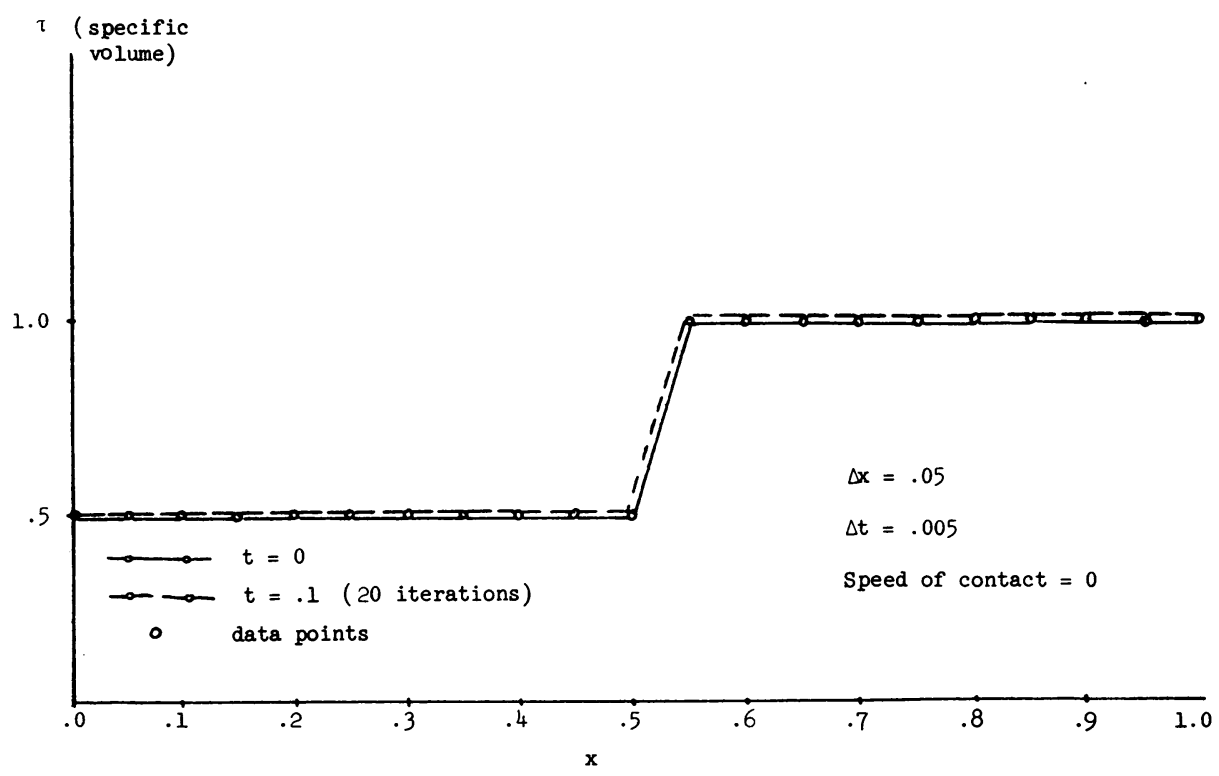

FIGURE 3

Lagrange equations $-1 \mathrm{D}$ nonisentropic

0 -speed contact discontinuity

(Initial data is steady solution)

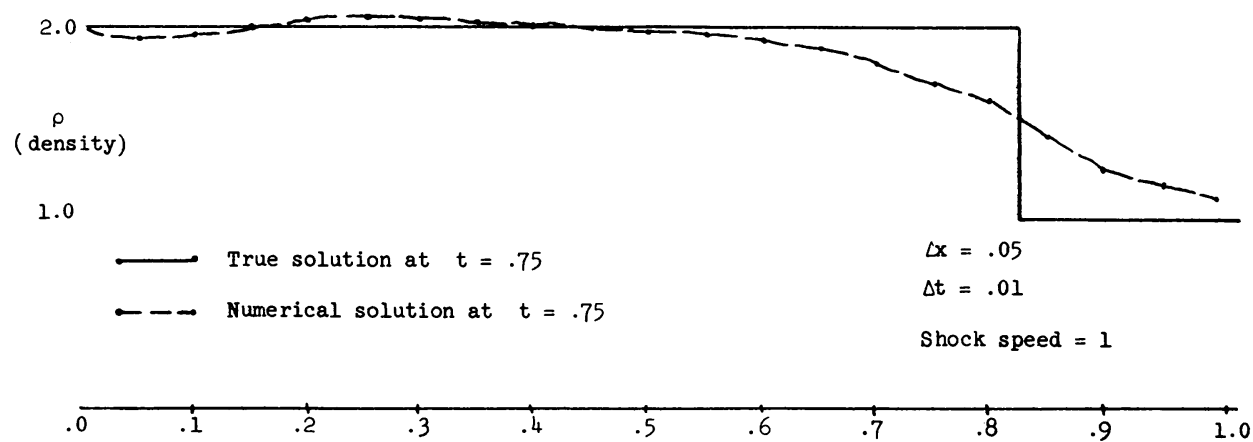

FIGURE 4

Euler equations-1D nonisentropic

Moving shock

(Initial data is that of moving shock with random noise perturbation) 


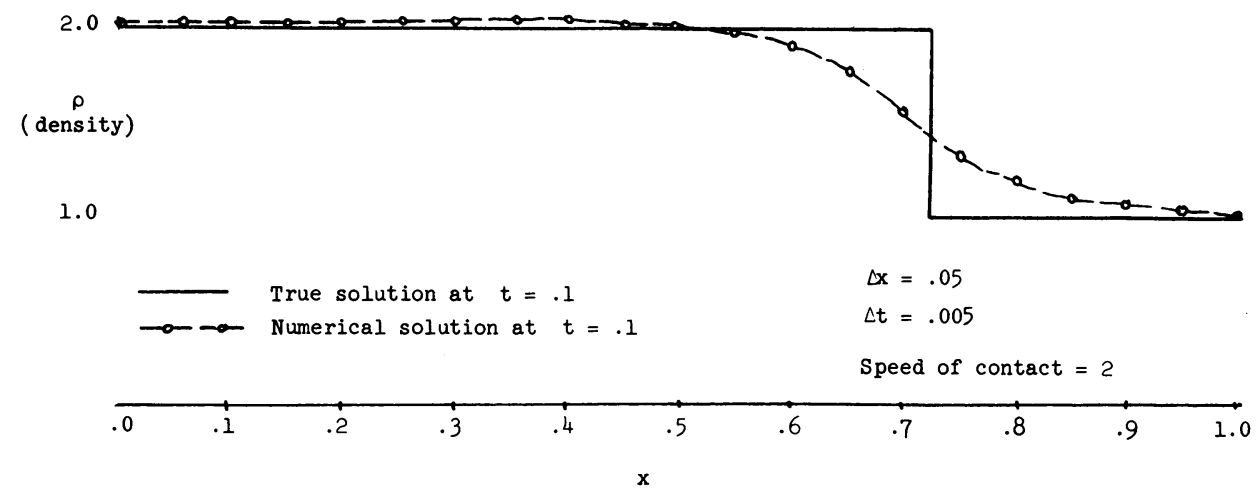

FIGURE 5

Euler equations-1D nonisentropic

Moving contact discontinuity

(Initial data is that of contact with no perturbation)

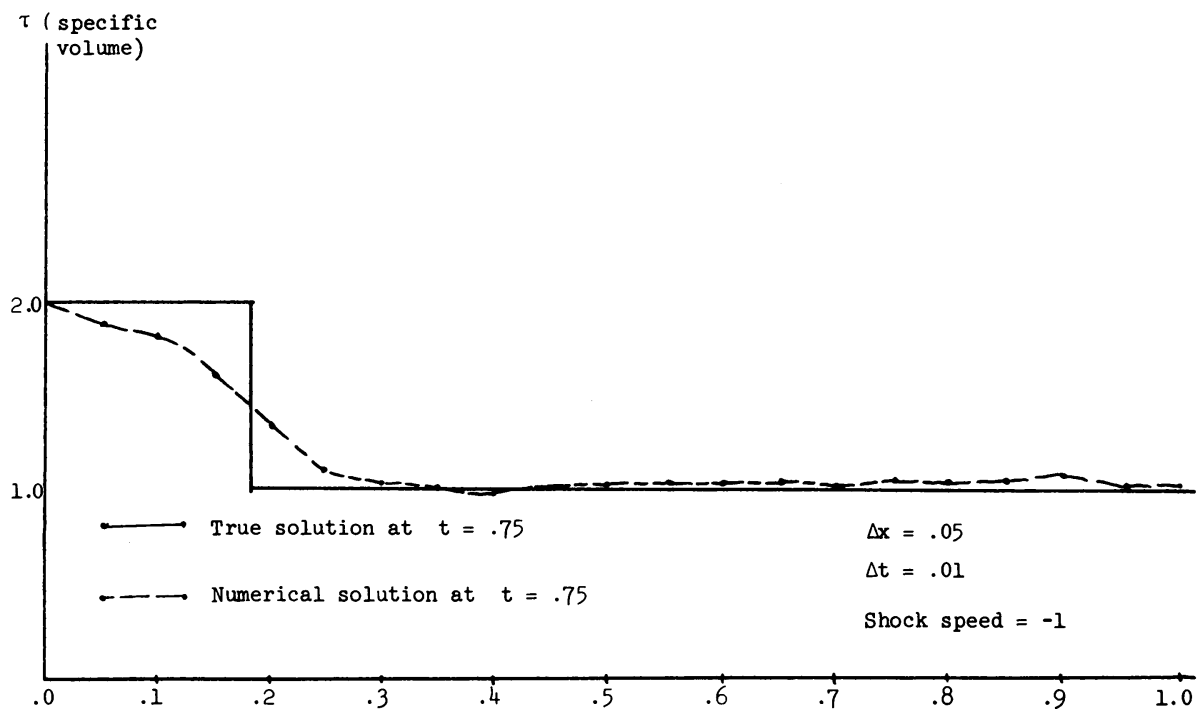

FIGURE 6

Lagrange equations-1D nonisentropic

Moving shock

(Initial data is that of moving shock with random noise perturbation) 
In cases 2 and 3 the initial data given was that of the steady solution. In the case of the 0 -speed shock, however, the initial data was a perturbation of a steady shock as seen in Figure 1. In all three cases the scheme is seen to yield sharp profiles with the profiles for the contacts being exact (a property of this scheme). Further the results in Figure 1 indicate stability for shock profiles as the perturbation is seen to settle down to that of the steady solution.

We next display scheme performance with respect to moving discontinuities. Here again are three examples:

1. (Figure 4) Moving shock-Euler equations 1-D nonisentropic ( $\rho$ component).

2. (Figure 5) Moving contact-Euler equations 1-D nonisentropic ( $\rho$ component).

3. (Figure 6) Moving shock-Lagrange equations 1-D nonisentropic ( $\tau$ component).

The initial data given in each case was two constant states $\left(w_{L}\right.$ and $\left.w_{R}\right)$ chosen so as to generate the desired moving discontinuity. In the two shock cases perturbations similar to those shown in Figure 1 were applied to the initial data. The results in the figures are then the numerical solutions after a large number of iterations plotted against the analytic solution. They tend to indicate smearing. This smearing occurs over a range of 5-7 points. This is not surprising, since all first-order upwind schemes are highly dissipative away from sonic points.

Next we consider the scheme in two space dimensions. We take a discontinuity which is oblique with respect to the $x$ and $y$ coordinate axes. The scheme was implemented for the 2-D isentropic Euler equations. The scheme was given as initial data a steady shock oriented at various angles with respect to the $x$-axis $-0^{\circ}, 15^{\circ}$, $30^{\circ}, 45^{\circ}$. We prescribe numerical boundary conditions which are the exact values of $w$ for such a skew-steady shock. After 20 iterations, $(t=.1$ seconds), the degree of profile sharpness that was preserved was viewed and is displayed in Figures 7,8,9 and 10. In the left half of each figure is shown the profile at $t=0$ while the right half displays the profile after the 20 iterations. The results are digitized and the following table provides the appropriate key:

\begin{tabular}{c|c}
\hline Digit & Value of $\rho$ \\
\hline 0 & $.50 \leqslant \rho<.55$ \\
1 & $.55 \leqslant \rho<.60$ \\
2 & $.60 \leqslant \rho<.65$ \\
3 & $.65 \leqslant \rho<.70$ \\
4 & $.70 \leqslant \rho<.75$ \\
5 & $.75 \leqslant \rho<.80$ \\
6 & $.80 \leqslant \rho<.85$ \\
7 & $.85 \leqslant \rho<.90$ \\
8 & $.90 \leqslant \rho<.95$ \\
9 & $.95 \leqslant \rho<1.00$ \\
\hline
\end{tabular}

The figures demonstrate a good degree of profile sharpness being maintained for all orientation angles. Indeed within any row or column the number of grid points taking digital values strictly between 0 and 9 is at most 5 and often 4 or 3 . Moreover, this number is at most 2 in the direction normal to the shock. 
000000000099999999999 000000000099999999999 000000000099999999999 000000000099999999999 000000000099999999999 000000000099999999999 000000000099999999999 000000000099999999999 000000000099999999999 000000000099999999999 000000000099999999999 000000000099999999999 000000000099999999999 000000000099999999999 00.0000000099999999999 000000000099999999999 000000000099999999999 000000000099999999999 000000000099999999999 000000000099999999999 000000000099999999999

$$
t=0
$$

000000000099999999999 000000000199999999999 000000000199999999999 000000000199999999999 000000000199999999999 000000000199999999999 000000000199999999999 000000000199999999999 000000000199999999999 000000000199999999999 000000000199999999999 000000000199999999999 000000000199999999999 000000000199999999999 000000000199999999999 000000000199999999999 000000000199999999999 000000000199999999999 000000000199999999999 000000000199999999999 000000000099999999999 $t=.1$ (20 1terations)

FIGURE 7

Euler equations 2-D isentropic Shock orientation angle $-0^{\circ}$

000000000099999999999 000000000999999999999 000000000999999999999 000000000999999999999 000000000999999999999 000000009999999999999 000000009999999999999 000000009999999999999 000000099999999999999 000000099999999999999 000000099999999999999 000000099999999999999 000000999999999999999 000000999999999999999 000000999999999999999 000000999999999999999 ) 00009999999999999999 000009999999999999999 000009999999999999999 000099999999999999999 000099999999999999999

$$
\mathrm{t}=\mathrm{o}
$$

000000000099999999999 000000000599999999999 000000001799999999999 000000002999999999999 000000004999999999999 000000006999999999999 000000028999999999999 000000039999999999999 000000069999999999999 000000179999999999999 000000299999999999999 000000499999999999999 000000699999999999999 000001799999999999999 000002999999999999999 000004999999999999999 000006999999999999999 000028999999999999999 000049999999999999999 000079999999999999999 000099999999999999999 $t=.1$ (20 1terations)

Figure 8 


\begin{tabular}{|c|c|}
\hline 000000000099999999999 & 00000000009999999999 \\
\hline 000000000099999999999 & 00000000039999999999 \\
\hline 000000000999999999999 & 00000000179999999999 \\
\hline 000000000999999999999 & 00000000489999999999 \\
\hline 000000009999999999999 & 00000001799999999999 \\
\hline 000000009999999999999 & 00000004999999999999 \\
\hline 0000000999999 & 00000037999999999999 \\
\hline 0000009999999999 & 00000168999999999999 \\
\hline 000000999999999999999 & 00000489999999999999 \\
\hline 000009999999999999999 & 00001799999999999999 \\
\hline 000009999999999999999 & 00004899999999999999 \\
\hline 000099999999999999999 & 00017999999999999999 \\
\hline 000099999999999999999 & 00049999999999999999 \\
\hline 000999999999999999999 & 00379999999999999999 \\
\hline 009999999999999999999 & 01689999999999999999 \\
\hline 009999999999999999999 & 03899999999999999999 \\
\hline 099999999999999999999 & 06999999999999999999 \\
\hline 099999999999999999999 & 08999999999999999999 \\
\hline 999999999999999999999 & 99999999999999999999 \\
\hline 999999999999999999999 & 99999999999999999999 \\
\hline $\begin{array}{c}999999999999999999999 \\
t=0\end{array}$ & $\begin{array}{c}99999999999999999999 \\
t=.1 \quad(20 \text { 1terations })\end{array}$ \\
\hline
\end{tabular}

Figure 9

Euler equations 2-D isentropic Shock orientation angle $-30^{\circ}$

000000000000099999999

000000000000999999999 000000000009999999999 000000000099999999999 000000000999999999999 000000009999999999999 000000099999999999999 000000999999999999999 000009999999999999999 000099999999999999999 000999999999999999999 009999999999999999999 099999999999999999999 999999999999999999999 999999999999999999999 999999999999999999999 ?99999999999999999999 999999999999999999999 999999999999999999999 999999999999999999999 999999999999999999999

$$
t=0
$$

000000000000099999999 000000000003799999999 000000000037999999999 000000000379999999999 000000003799999999999 000000037999999999999 000000379999999999999 000003799999999999999 000037999999999999999 000379999999999999999 003799999999999999999 037999999999999999999 079999999999999999999 999999999999999999999 999999999999999999999 999999999999999999999 999999999999999999999 999999999999999999999 999999999999999999999 999999999999999999999 999999999999999999999

$$
t=.1 \text { (20 1terations) }
$$

FIgURE 10 


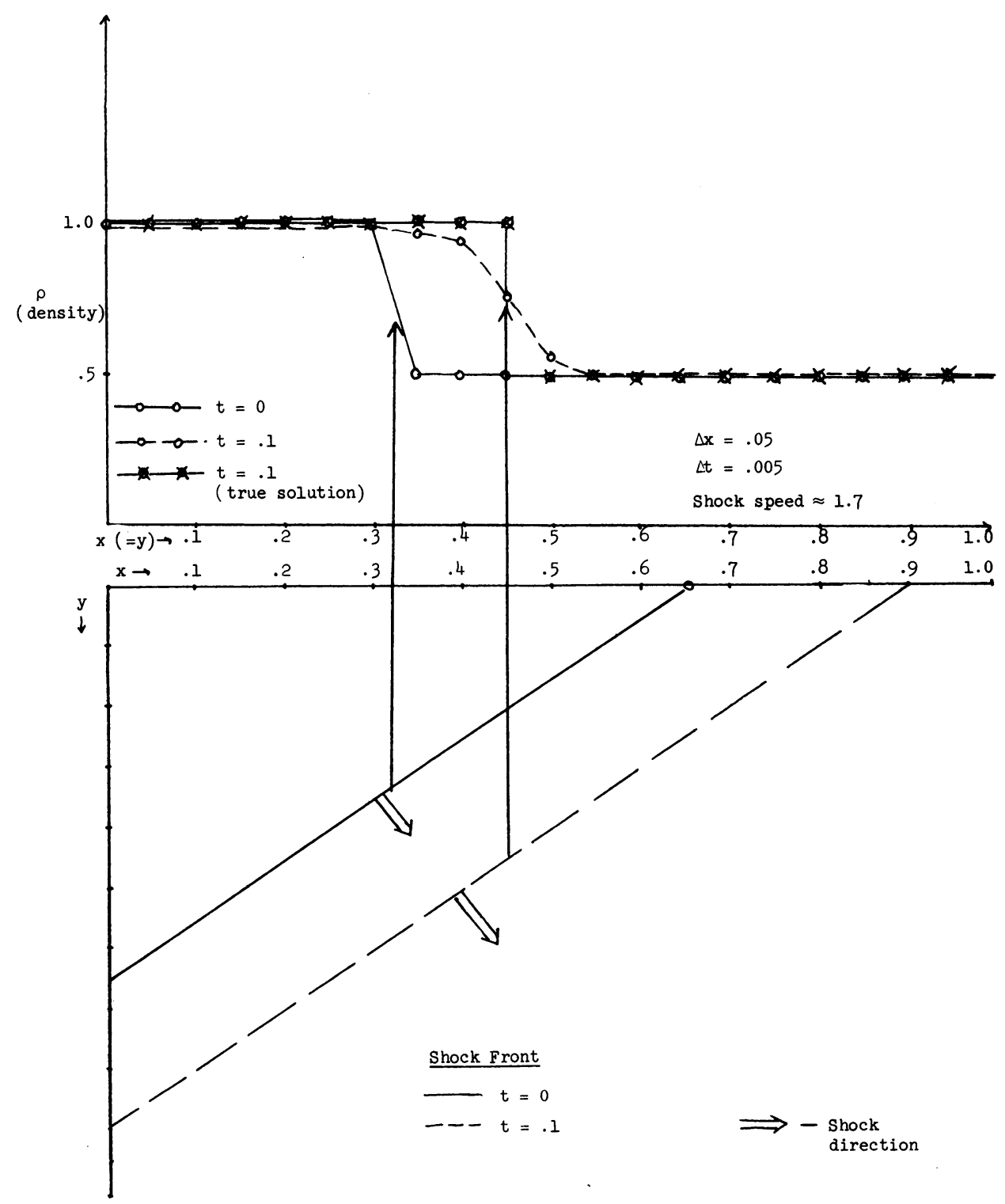

FIGURE 11

Euler equations-2D isentropic

Shock moving at $45^{\circ}$ angle $s \approx 1.7$

Profile of $\rho(x, y)$ along line $x=y$

We also ran the $30^{\circ}$ shock case for 100 iterations with no deterioration in profile sharpness. (Not displayed here.)

Two further cases were run for the 2-D Euler equations:

1. (Figure 11) Euler equations-2D isentropic shock moving at $45^{\circ}$ angle, shock speed $=1.7$. 


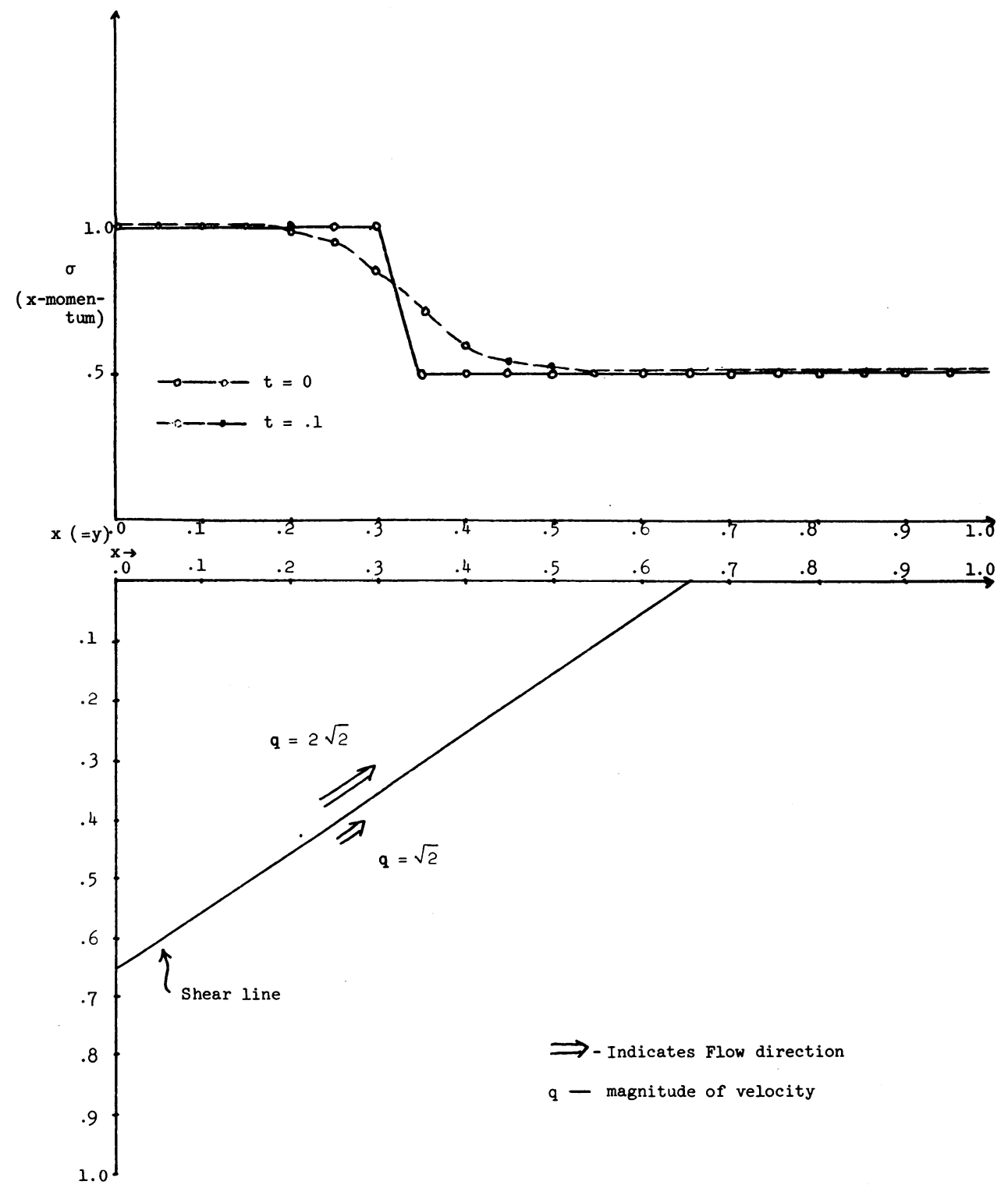

FIGURE 12

Euler equations-2D isentropic

Shear flow at $45^{\circ}$ angle

Profile of $\sigma(x, y)$ along line $x=y$

$\sigma=\rho u$ is the $x$-momentum

2. (Figure 12) Euler equations-2D isentropic shear flow at $45^{\circ}$ angle.

In the top half of each figure is shown a cross section of the profile along the line $x=y$ both at $t=0$ and $t=.1$ (20 iterations). In the bottom half is shown the view when looking straight downward again at $t=0$ and $t=.1$. 
Neither the shear flow nor the moving shock appear to enjoy the same sharpness as the steady shocks (Figures 7-10). After the usual 20 iterations both displayed a fair degree of smearing.

Finally we discuss scheme stability as a function of the Courant number. In this regard we consider again the 0 -speed shock case of Figure 1 . As before, the initial data was that of the steady solution with a perturbation applied. Five cases were run $(\mu(\Delta t / \Delta x)=.1, .2, .4, .8$, and 1.6). The scheme showed to be stable for 100 iterations for the cases $\mu=.1, .2$, and .4 , but was unstable for $\mu=.8$ and 1.6. According to linearized stability theory the theoretical range for the scheme to be stable is $\mu \leqslant 1 / \rho(A)$ where $A$ is the Jacobian of $f . \rho(A)$ for this case turns out to be $\approx 1.65$ which translates into $\lambda \leqslant .61$. Thus the numerical results agree with the theory.

Department of Mathematics

University of California, Los Angeles

Los Angeles, California 90024

1. B. ENGquist \& S. OSher, "Stable and entropy satisfying approximations for transonic flow calculations," Math. Comp., v. 34, 1980, pp. 45-75.

2. B. Engquist \& S. Osher, "One sided difference schemes and transonic flow," Proc. Nat. Acad. Sci. U.S.A., v. 77, 1980, pp. 3071-3074.

3. B. ENGQuist \& S. OSHER, "One sided difference approximations for nonlinear conservation laws," Math. Comp., v. 36, 1981, pp. 321-352.

4. B. ENGQUIST \& S. OSHER, Upwind Difference Equations for Systems of Conservation Laws-Potential Flow Equations, MRC Technical Report \#2186, Univ. of Wisconsin, 1981.

5. K. O. Friedrichs \& P. D. LAX, "Systems of conservation laws with a convex extension," Proc. Nat. Acad. Sci. U.S.A., v. 68, 1971, pp. 1686-1688.

6. S. K. Godunov, "A finite-difference method for the numerical computation of discontinuous solutions of the equations of fluid dynamics," Mat. Sb., v. 47, 1959, pp. 271-290. (Russian)

7. P. M. GoORJian \& R. VAN Buskirk, Implicit Calculations of Transonic Flow Using Monotone Methods, AIAA-81-0331, 1981.

8. A. Harten, J. M. Hyman \& P. D. Lax, "On finite difference approximations and entropy conditions for shocks," Comm. Pure Appl. Math., v. 29, 1976, pp. 297-322.

9. A. Harten, P. D. LAX \& B. van LeER, "On upstream differencing and Godunov type schemes for hyperbolic conservation laws." (Preprint.)

10. G. Jennings, "Discrete shocks," Comm. Pure Appl:' Math., v. 27, 1974, pp. 25-37.

11. N. N. KuZNETSOv, "On stable methods for solving non-linear first order partial differential equations in the class of discontinuous functions," Topics in Numerical Analysis II (Proc. Roy. Irish Acad. Conf. on Numerical Analysis, 1976), Ed. J. J. H. Miller, Academic Press, New York, 1977, pp. 183-197.

12. P. D. LAx, Hyperbolic Systems of Conservation Laws and the Mathematical Theory of Shock Waves, SIAM Regional Conf. Series Lectures in Appl. Math. No. 11, SIAM, Philadelphia, Pa., 1973.

13. P. D. LaX \& B. Wendroff, "Systems of conservation laws," Comm. Pure Appl. Math., v. 13, 1960, pp. 217-237.

14. A. Majda \& S. Osher, "Numerical viscosity and the entropy condition," Comm. Pure Appl. Math., v. 32, 1979, pp. 797-838.

15. A. Majda \& J. Ralston, "Discrete shock profiles for systems of conservation laws," Comm. Pure Appl. Math., v. 22, 1979, pp. 445-482.

16. M. S. Mock, "Some higher-order difference schemes enforcing an entropy inequality," Michigan Math. J., v. 25, 1978, pp. 325-344.

17. E. M. MURman, “Analysis of embedded shockwaves calculated by relaxation methods," AIAA J., v. 12, 1974, pp. 626-633.

18. S. OSHER, "Approximation par éléments finis avec décentrage pour des lois de conservation hyperboliques nonlinéares multi-dimensionelles," C.R. Acad. Sci. Paris, Sér. A, v. 290, 1980, pp. 819-821. 
19. S. OsHer, Numerical Solution of Singular Perturbation Problems and Hyperbolic Systems of Conservation Laws, Math. Studies No. 47 (0. Axelsson, L. S. Frank, A. van der Sluis, Eds.), North-Holland, Amsterdam, 1981, pp. 179-205.

20. S. OSHER, "Nonlinear singular perturbation problems and one-sided difference schemes," SIAM J. Numer. Anal., v. 18, 1981, pp. 129-144.

21. S. OSHER, B. ENGQUIST \& M. W. MANN, "Upwind difference schemes for the potential equation of transonic flow." (In preparation.)

22. P. L. RoE, "Approximate Riemann solvers, parameter vectors, and difference schemes," J. Comput. Phys. (To appear.)

23. B. VAN LEER, Upwind Differencing for Hyperbolic Systems of Conservation Laws, ICASE Internal Report Document \# 12, 1980. 